\title{
UNIFORM ASYMPTOTIC FORMULAE FOR FUNCTIONS WITH TRANSITION POINTS
}

\author{
BY \\ T. M. CHERRY
}

1. Introduction. Let $g(z, w)$ be an analytic function, regular at $z=0, w=0$. We are to investigate asymptotic formulae for the solutions of the differential equation

$$
\frac{d^{2} y}{d z^{2}}+y\left\{-\nu^{2} z+g\left(z, \nu^{-2}\right)\right\}=0
$$

where $\nu$ is a large parameter. More, precisely, we desire approximations whose error is $O\left(\nu^{-m}\right)$, uniformly for $z$ in a closed region, independent of $\nu$, having the point $z=0$ in its interior or on its boundary, $m$ being an arbitrarily large integer.

The investigation will cover the solutions, in the neighbourhood of $x=0$, of the more general equation

$$
\frac{d^{2} Y}{d x^{2}}+R\left(x, \nu^{-2}\right) \frac{d Y}{d x}+Y\left\{-\nu^{2} f(x)+Q\left(x, \nu^{-2}\right)\right\}=0,
$$

in which $R(x, w), Q(x, w)$ are regular at $x=0, w=0$ and $f(x)$ has a simple zero at $x=0$; for (see $\$ 2$ ) this may be reduced to the form (1.1) by a change of variables, regular at $x=z=0$. When all the symbols denote real numbers, the solutions of (1.2) are monotonic or oscillating according as $\nu^{2} f-Q$ $+4^{-1} R^{2}+2^{-1} d R / d x$ is positive or negative; and when $\nu$ is large, this quantity changes sign at a point near $x=0$, on account of the simple zero of $f(x)$ at $x=0$. Thus as $x$ passes through 0 the solutions change from monotonic to oscillating, and we may call $x=0$ a transition point $\left.{ }^{1}\right)$. It is this transition which distinguishes our problem from the simpler one in which the $x$-region includes no zero of $f(x)$.

The guiding idea of the investigation is familiar: "approximately identical differential equations have approximately identical solutions." A significant approximation to (1.1) will be an equation having the same features when $z$ is near 0. Accordingly the project stated in the first paragraph is crystallized: the approximations to solutions of (1.1) are to be solutions of a differential equation of the same form as (1.1).

The simplest equation of this form is the Airy equation

Presented to the Society, February 26, 1949; received by the editors January 13, 1949 and, in revised form, September 13, 1949.

(1) In the nature of the case there is no single point at which a solution changes character, and there is no definition of "transition point" which is invariant under change of variables. Our use of the term will be essentially descriptive, to emphasize that such and such a point is about the centre of the neighbourhood which is of primary interest. 


$$
\frac{d^{2} \eta}{d \zeta^{2}}-\nu^{2} \zeta \eta=0
$$

whose general solution is $\left({ }^{2}\right)$

$$
\eta=C \mathrm{Ai}\left(\nu^{2 / 3} \zeta\right)+D \mathrm{Ai}\left(\nu^{2 / 3} e^{ \pm 2 \pi i / 3} \zeta\right) .
$$

It is a significant approximation to (1.1) if we take $\zeta=z$; and it is on the basis of this approximation that R. E. Langer [3] has established (under precisely defined conditions) approximations to solutions of $(1.1)$, in the form $\left({ }^{3}\right)$

$$
y(z)=C_{\nu} \mathrm{Ai}\left(\nu^{2 / 3} z\right)\left\{1+O\left(\nu^{-1}\right)\right\}+D_{\nu} \mathrm{Ai}\left(\nu^{2 / 3} e^{2 \pi i / 3} z\right)\left\{1+O\left(\nu^{-1}\right)\right\} .
$$

The Airy functions are not elementary, but they are acceptable as approximations since their properties are well known and they are tabulated [2].

In the present paper a procedure is given for finding approximations which resemble (1.5), but in which the error is $O\left(\nu^{-2 n-1}\right)$, where $n$ is as large as we please. The underlying idea is that we must first find a differential equation, with a known solution, whose discrepancy from (1.1) is of order $\nu^{-2 n} y$. This is obtained (see §3) from (1.3) by means of a transformation,

$$
\zeta=\phi(z)=z+\nu^{-2} \phi_{1}(z)+\nu^{-4} \phi_{2}(z)+\cdots, \quad \eta=y\left(\phi^{\prime}(z)\right)^{1 / 2},
$$

in which the functions $\phi_{1}, \phi_{2}, \cdots$ are successively determined by elementary processes, and all are regular at $z=0$. By a familiar process we then ( $\$ 4)$ compare the solutions of this transform of (1.3) with those of (1.1).

The results may be presented in two forms, (i) and (ii). Examples are:

$$
s^{1 / 2} J_{\nu}\left\{\nu\left(1-s^{2}\right)^{1 / 2}\right\}=\left(\frac{2}{\phi^{\prime}(z)}\right)^{1 / 2} \cdot \frac{z^{1 / 4} \mathrm{Ai}\left\{\nu^{2 / 3} \phi(z)\right\}}{\nu^{1 / 3}}\left\{1+O\left(\nu^{-2 n-1}\right)\right\},
$$

where $z=\{(3 / 2)(\operatorname{arctanh} s-s)\}^{2 / 3}$ and $\phi(z)$ is given by a series (1.6) in which $\phi_{1}, \phi_{2}, \cdots$ are determined by one rule as far as $\phi_{n}$, and thence onwards by a different rule (see $\$ 3.2)$. For $|\arg \nu| \leqq \pi / 2$ and $\left|\arg \left(1-s^{2}\right)^{1 / 2}\right| \leqq \pi / 2$ this gives uniform approximation to $s^{1 / 2} J_{\nu}$ except in small neighbourhoods of the zeros of the function, where a slightly modified formula is required.

$$
\begin{aligned}
s^{1 / 2} J_{\nu}\left\{\nu\left(1-s^{2}\right)^{1 / 2}\right\}= & 2^{1 / 2} \nu^{-1 / 3} z^{1 / 4}\left[\mathrm { Ai } ( \nu ^ { 2 / 3 } z ) \left\{1+q_{2} \nu^{-2}+\cdots\right.\right. \\
& \left.+q_{2 n} \nu^{-2 n}+O\left(\nu^{-2 n-2}\right)\right\} \\
& +\nu^{-1 / 3} \mathrm{Ai}^{\prime}\left(\nu^{2 / 3} z\right)\left\{q_{1} \nu^{-1}\right. \\
& +\cdots \\
& \left.\left.+q_{2 n-1} \nu^{-2 n+1}+O\left(\nu^{-2 n-1}\right)\right\}\right],
\end{aligned}
$$

(2) The notation here used for Airy functions was introduced by H. Jeffreys [1]; the definition of $\operatorname{Ai}(x)$ in terms of Bessel functions of order 1/3 is given in equations (4.8), (4.23) of the present paper. For tables of the functions, and an account of their properties, see [2]. Numbers in brackets refer to the references cited at the end of the paper.

(3) Langer's results are here expressed in Airy-function notation. 
where $q_{1}, q_{2}, \cdots$ are determined by one rule throughout. This is analogous to familiar elementary asymptotic series; its region of validity includes the zeros of $J_{v}$, but $s$ must be so restricted that $z$ is bounded.

The general results are reached in $\$ \S 4.6,4.7$, and the Bessel-function formulae, from which the preceding have been quoted, are in $\$ \S 4.8,4.9$.

In $\$ 5$ are indicated the formulae for approximating to certain hypergeometric functions $F\left(a_{\nu}, b_{\nu} ; \nu+1 ; \tau\right)$ of large order $\nu$ by means of Bessel functions of order $\nu$. This type of approximation is chosen for reasons of functional similarity, and for both theoretical and practical purposes is as acceptable as the Airy-function approximation. Here we find a single formula, (5.11), which covers all large values of $\nu$ (without restriction on arg $\nu$ ) and all values of $\tau$ apart from a neighbourhood of the cut $1 \leqq \tau<+\infty$, and small neighbourhoods of the zeros of $F\left(a_{v}, b_{v} ; \nu+1 ; \tau\right)$.

The theory has not been carried to the point of evaluating the constants of the error-terms, so numerical comparişons of exact against approximate values are of interest. Such checks are referred to in $\$ 4.8$; suffice it here to say that, for the purposes of (1.7), 2 is a "large number," for the formula (with $n=2$ ) gives $J_{2}(x)$ from $x=0.6$ to 20 (the range examined) correct to 1 in 30000 . The formulae of $\$ 5$ are not so spectacular; for $|\nu| \geqq 8.5$ the accuracy (with $n=2$ ) is about 1 in a million.

To economize space it has been necessary to suppress a good deal of detail: and algebraic detail merges into logical detail; it is hoped however that no logical point of any substance has been treated too summarily.

Notation. As usual, certain symbols are used generically, namely, $A$ (constants), $O$ (Landau's order symbol), $P$ (power series, convergent when the arguments are sufficiently small), $\epsilon$ (positive number, arbitrarily small). Certain other symbols have different significations in different transient contexts. For a given choice of datum-functions the $A$ 's and $O$ 's are absolute, except as stated in the context; and except that, throughout, the constant implied by $O\left(\nu^{-2 n-1}\right)$ depends on $n .|\nu|$ is usually supposed, and on key occasions is stated, to be "sufficiently large." Arguments of functional symbols are often suppressed, or only the one which is at the moment important may be shown.

2. The canonic forms of differential equation. Consider a linear differential equation of the second order, in the normal form (2.1) below. We can make an arbitrary change of independent variable, and shall preserve the normal form provided we make also a suitable change of dependent variable:

Lemma 1. The transformation $z=\phi(u), y=w\left(\phi^{\prime}(u)\right)^{1 / 2}$, applied to the differential equation

$$
\frac{d^{2} y}{d z^{2}}+y F(z)=0
$$


gives

$$
\frac{d^{2} w}{d u^{2}}+w\left\{F(\phi(u))\left(\phi^{\prime}(u)\right)^{2}+\frac{\phi^{\prime \prime \prime}(u)}{2 \phi^{\prime}(u)}-\frac{3}{4}\left(\frac{\phi^{\prime \prime}(u)}{\phi^{\prime}(u)}\right)^{2}\right\}=0 .
$$

This result is well known, and its proof is trivial.

If in (1.2) we replace $Y$ by $y \exp \left(-2^{-1} \int R d x\right)$ we obtain a similar equation with $R, Q$ replaced by $0, Q-R^{2} / 4-2^{-1} d R / d x$; and the latter function has the form $P\left(x, \nu^{-2}\right)$ since $R, Q$ have this form. Now put $x=\phi(u), y=w\left(\phi^{\prime}(u)\right)^{1 / 2}$, with $\phi(u)$ so chosen that $\left(\phi^{\prime}(u)\right)^{2} f(x)=1$, with $u=0$ for $x=0$. Since, by hypothesis, $f(x)=a_{1} x+a_{2} x^{2}+\cdots$ with $a_{1} \neq 0$, we obtain

$$
u=\frac{2}{3} a_{1}^{1 / 2} x^{3 / 2}\{1+x P(x)\} .
$$

Thence $x=\phi(u)$ is a power series in $u^{2 / 3}$, and an easy calculation of the terms in (2.2) gives the form

$$
\frac{d^{2} w}{d u^{2}}+w\left\{-\nu^{2}+\frac{5}{36 u^{2}}+W\left(u, \nu^{-2}\right)\right\}=0,
$$

with

$$
W\left(u, \nu^{-2}\right)=u^{-2 / 3} P\left(u^{2 / 3}, \nu^{-2}\right) .
$$

Conversely, the substitution

$$
u=\phi(z)=\frac{2}{3} z^{3 / 2}, \quad w=y \phi^{\prime 1 / 2}=y z^{1 / 4}
$$

converts (2.3) into

$$
\frac{d^{2} y}{d z^{2}}+y\left\{-\nu^{2} z+g\left(z, \nu^{-2}\right)\right\}=0
$$

with

$$
g\left(z, \nu^{-2}\right)=z W=P\left(z, \nu^{-2}\right) .
$$

Our problem concerning approximations to the solutions of (1.2) is thus reduced to a similar problem concerning solutions of $(2.3)$ or $(2.5)$, in the neighbourhood of the transition-point $u=0$ or $z=0$. We describe (2.3) as in canonic form $\mathrm{B}$ and (2.5) as in canonic form $\mathrm{A}\left({ }^{4}\right)$. In view of the transformation (2.4), we can at pleasure work with either. Near the transition-point the A-form is simpler, and we shall use it in the transformation theory of $\S 3$. But the B-form is simpler when $z, u$ are not small, and we shall use it for the asymptotic theory of $\S 4$. It appears moreover (Examples 2, 3 below) that for equations of the hypergeometric family, and others (for example, Mathieu's

(4) $\mathrm{B}=$ Bessel, cf. (2.6); A=Airy. 
equation), the canonic A-form is further removed from the original differential equation than is the B-form, and that the latter is analytically the simpler. When such equations are in question it may therefore be preferable to use the B-form throughout; this procedure is illustrated in $\$ 5$ and in Cherry [4].

Example 1. The Airy equation (1.3) is in canonic form A. The corresponding B-form is

$$
\frac{d^{2} w}{d u^{2}}+w\left(-\nu^{2}+\frac{5}{36 u^{2}}\right)=0,
$$

and the general solution of this is

$$
w=C L(\nu u)+D L\left(\nu u e^{\pi i}\right),
$$

where

$$
L(x)=x^{1 / 2} K_{1 / 3}(x),
$$

$K_{1 / 3}$ being the Bessel function so denoted.

EXAMPLE 2. Bessel's equation of order $\nu$ and argument $\lambda\left(1-s^{2}\right)^{1 / 2}$. The reduction indicated at the beginning of this section gives the differential equation

$$
\frac{d^{2} w}{d u^{2}}+w\left(-\nu^{2}+\frac{5}{36 u^{2}}+W\right)=0
$$

where

$$
\begin{aligned}
& u=\operatorname{arctanh} s-s \\
& W=\left(\frac{1}{s^{2}}-1\right)\left(\frac{5}{4 s^{4}}-\frac{1}{4 s^{2}}+\lambda^{2}-\nu^{2}\right)-\frac{5}{36 u^{2}},
\end{aligned}
$$

has the general solution

$$
w=C s^{1 / 2} J_{\nu}\left\{\lambda\left(1-s^{2}\right)^{1 / 2}\right\}+D s^{1 / 2} Y_{\nu}\left\{\lambda\left(1-s^{2}\right)^{1 / 2}\right\} .
$$

So far as concerns approximations to Bessel functions of large order and argument we can put $\lambda=\nu$, but the wider assumption $\lambda^{2}-\nu^{2}=\Phi\left(\nu^{-2}\right)$ leads, as will be seen in $\$ 5$, to useful generality. By reversing the expansion $u=(1 / 3) s^{3}$ $\cdot\left(1+3 s^{2} / 5+\cdots\right)$ for the neighbourhood of $u=0$ we find

$$
\begin{aligned}
W= & \left(\lambda^{2}-\nu^{2}-\frac{1}{35}\right)\left(\frac{1}{(3 u)^{2 / 3}}-\frac{3}{5}+\frac{2}{35}(3 u)^{2 / 3}+\cdots\right) \\
& -\left(\frac{23}{525}+\frac{36}{25 \cdot 49 \cdot 11}(3 u)^{2 / 3}+\cdots\right)
\end{aligned}
$$

and the form (2.3) is verified. 
The Riemann surface for $W$ as a function of $u$ has infinitely many sheets, of which we need consider only the three which are cyclically connected round $u=0$. The fundamental unit (Fig. 1 (iii)) is the sector $0 \leqq \arg u \leqq 3 \pi / 2$ with a cut from $u=i \pi$ to $i \infty$, and by reflections in the boundary rays through $u=0$ we get in all four such units which together form the three sheets. We convert (2.7) into form A by putting $u=2 z^{3 / 2} / 3, w=y z^{1 / 4}$, and to the part of the $u$-surface for which $|\arg u| \leqq 3 \pi / 2$ corresponds a schlicht $z$-plane with three cuts (Fig. 1 (iv)); but since $g=z W$ and $W\left(u e^{3 \pi i}\right)=W(u), g$ is regular across the cut from $z=0$ to $-\infty$, and this cut can be sealed up. In Fig. 1 we see also that in sealing this cut we seal also a cut in the plane of $x=\left(1-s^{2}\right)^{1 / 2}$ from $x=1$ to $+\infty$.

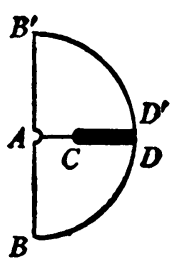

(i) $x$-plane; $x=\left(1-s^{2}\right)^{1 / 8}$

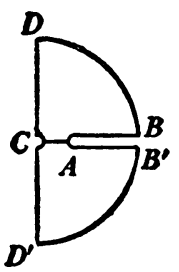

(ii) s-plane

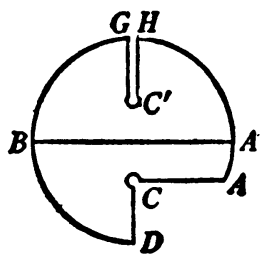

(iii) $u$-plane; $u=\operatorname{arctanh} s-s$

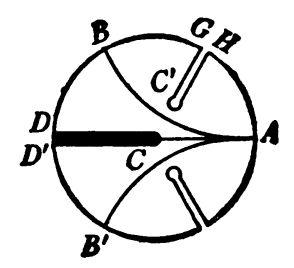

(iv) z-plane; $z=\left(\frac{3}{2} u\right)^{2 / 2}$

Fig. 1

Black area: Sealed-up cuts.

$$
\begin{aligned}
& \text { Point } A: \quad x=0, \quad s=1, \quad u=+\infty, \quad z=+\infty \text {. } \\
& C: \quad x=1, \quad s=0, \quad u=0, \quad z=0 \\
& D: \quad x=+\infty, \quad s=i \infty, \quad u=\infty e^{3 \pi i / 2}, \quad z=-\infty \text {. } \\
& B: \quad x=-i \infty, \quad s=+\infty, \quad u=\infty e^{\pi i}, \quad z=\infty e^{2 \pi i / 3} \text {. } \\
& C^{\prime}: \quad u=\pi i, \quad z=(3 \pi / 2)^{2 / 3} e^{\pi i / 3} \text {. }
\end{aligned}
$$

Note: In the $u$-plane, the reflection in $C A$ has been omitted.

For later reference we note the forms of $W, g$ in the two infinite sectors of the cut $z$-plane; these are easily found from the appropriate local approximations to the $s u$-relation (2.8): For $|\arg z| \leqq \pi / 3-\epsilon$,

$$
\begin{aligned}
W & =-\frac{5}{36 u^{2}}+4\left(1+\lambda^{2}-\nu^{2}\right) e^{-2-2 u}+\cdots \\
& =-\frac{5}{36 u^{2}}+e^{-2 u} P\left(e^{-2 u}, \nu^{-2}\right), \\
g & =z W=O\left(z^{-2}\right) .
\end{aligned}
$$

For $|\arg (-z)| \leqq 2 \pi / 3$,

$$
\begin{aligned}
& W=\nu^{2}-\lambda^{2}+\left(\frac{1}{9}-\lambda^{2}-\nu^{2}\right) u^{-2}+\cdots=\nu^{2}-\lambda^{2}+u^{-2} P\left(u^{-1}\right), \\
& g=z W=\left(\nu^{2}-\lambda^{2}\right) z+O\left(z^{-2}\right) .
\end{aligned}
$$


Example 3. A hypergeometric equation. The equation

$$
\frac{d^{2} y}{d \tau^{2}}+\left(\frac{1}{\tau}-\frac{\beta}{1-\tau}\right) \frac{d y}{d \tau}+\nu^{2}\left(\frac{\beta}{2 \tau(1-\tau)}-\frac{1}{4 \tau^{2}}\right) y=0
$$

has as its general solution

$$
y=C \tau^{\nu / 2} F_{\nu}(\tau)+D \tau^{-\nu / 2} F_{-\nu}(\tau),
$$

where $F_{\nu}(\tau)$ denotes the hypergeometric function

$$
\begin{gathered}
F_{\nu}(\tau)=F\left(a_{\nu}, b_{\nu} ; \nu+1 ; \tau\right), \\
a_{\nu}+b_{\nu}=\nu+\beta, \quad a_{\nu} b_{\nu}=-\beta \nu(\nu-1) / 2 .
\end{gathered}
$$

This and related functions are of importance in the theory of compressiblefluid flow, where, when the fluid is air, the value of $\beta$ is about 2.5. When $\nu$ is real $F_{\nu}(\tau)$ is monotonic for $0<\tau<\tau_{\text {s }}$ and oscillating for $\tau_{\varepsilon}<\tau<1$, where

$$
\tau_{s}=1 /(1+2 \beta) \text {. }
$$

By putting

$$
\begin{gathered}
t=\left(\frac{1-\tau / \tau_{s}}{1-\tau}\right)^{1 / 2}, \quad u=\operatorname{arctanh} t-\tau_{s}^{-1 / 2} \operatorname{arctanh}\left(t \tau_{s}^{1 / 2}\right), \\
w=(1-\tau)^{\beta / 2} t^{1 / 2} y=(1-\tau)^{\beta / 2} t^{1 / 2}\left\{C \tau^{\nu / 2} F_{\nu}(\tau)+D \tau^{-\nu / 2} F_{-\nu}(\tau)\right\},
\end{gathered}
$$

we convert (2.13) into

$$
\begin{gathered}
\frac{d^{2} w}{d u^{2}}+w\left(-\nu^{2}+\frac{5}{36 u^{2}}+W_{1}\right)=0, \\
W_{1}=\frac{1-t^{2}}{4\left(1-\tau_{s}\right)^{2}}\left\{\frac{5}{t^{6}}-\frac{1+6 \tau_{s}}{t^{4}}+\frac{3-4 \tau_{s}}{t^{2}}\right. \\
\left.\quad+\left(1-2 \tau_{s}\right)\left(1-4 \tau_{s}\right)\right\}-\frac{5}{36 u^{2}} ;
\end{gathered}
$$

and this is readily verified to be in canonic form $\mathrm{B}$. Considering $W_{1}$ as a function of $u$, the fundamental unit of the Riemann surface is the same as for $W$ (Example 2), except that there is an additional branch point on $C D$, at $u=2^{-1} \pi\left(\tau_{s}^{-1 / 2}-1\right) e^{3 \pi i / 2}$, and the ray thence to $u=\infty e^{3 \pi i / 2}$ must be taken as a cut. The correspondence between $\tau, t, u, z$ is shown in Fig. 2 .

The form-A equation corresponding to (2.17) involves a function $g_{1}=z W_{1}$ which is regular in the same domain as $g$ (Example 2), except for a new cut from $z=-z_{1}$ to $-\infty, z_{1}=\left\{(3 \pi / 4)\left(\tau_{8}^{-1 / 2}-1\right)\right\}^{2 / 3}$; at $z=-z_{1}, g_{1}=\infty$. Near $\infty_{A}$,

$$
W_{1}=-5 / 36 u^{2}+e^{-2 u} P\left(e^{-2 u}\right), \quad g_{1}=O\left(z^{-2}\right) ;
$$

near $\infty_{B}$, 


$$
\begin{aligned}
W_{1} & =-\frac{\left(1-\tau_{s}\right)^{2}}{4 \tau_{s}}-\frac{5}{36 u^{2}}+e^{2 u \tau_{s}^{1 / 2}} P\left(e^{2 u \tau_{s}^{1 / 2}}\right), \\
g_{1} & =-\frac{\left(1-\tau_{s}\right)^{2}}{4 \tau_{s}} z+O\left(z^{-2}\right) .
\end{aligned}
$$

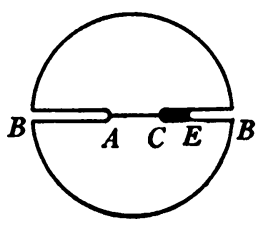

(i) T-plane

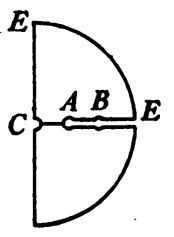

(ii) t-plane

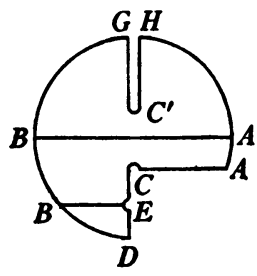

(iii) $u$-plane

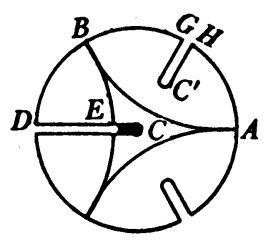

(iv) z-plane

Fig. 2

Black area: Sealed-up cuts.

Point $A: \quad \tau=0, \quad t=1, \quad u=+\infty, \quad z=+\infty$.

$C: \quad \tau=\tau_{s}, \quad t=0, \quad u=0, \quad z=0$.

$E: \quad \tau=1, \quad t=\infty, \quad u=u_{1} e^{8 \pi i / 2}, \quad z=-\left(3 u_{1} / 2\right)^{2 / 3} ; \quad u_{1}=2^{-1} \pi\left(\tau_{t}{ }^{-1 / 2}-1\right)$.

$B: \quad \tau=\infty, \quad t=\tau_{s}^{-1 / 2}, u=\infty e^{\pi i}, \quad z=\infty e^{2 \pi i / z}$.

Note: In the $u$-plane, the region $C A B E$ corresponds to the half-plane $\operatorname{Im} \tau<0$; the reflection in $C A$ has been omitted.

3. Approximate reduction of a canonic equation to the Airy equation. Let the transformation

$$
\zeta=\phi(z)=z+\sum_{1}^{\infty} \nu^{-2 r} \phi_{r}(z), \quad \quad \eta=y\left(\phi^{\prime}(z)\right)^{1 / 2}
$$

be applied to the Airy equation

$$
\frac{d^{2} \eta}{d \zeta^{2}}-\nu^{2} \zeta \eta=0
$$

By Lemma 1, the resulting equation would coincide exactly with the equation (2.5):

$$
\frac{d^{2} y}{d z^{2}}+y\left\{-\nu^{2} z+g\left(z, \nu^{-2}\right)\right\}=0
$$

provided

$$
\nu^{2} \phi(z)\left[\phi^{\prime}(z)\right]^{2}-\frac{\phi^{\prime \prime \prime}(z)}{2 \phi^{\prime}(z)}+\frac{3}{4}\left[\frac{\phi^{\prime \prime}(z)}{\phi^{\prime}(z)}\right]^{2}=\nu^{2} z-g\left(z, \nu^{-2}\right)
$$


This is a differential equation for $\phi(z)$, for which it is easy (see $\$ 3.2$ ) to find a series-solution in the form (3.1). It appears that this formal series is usually divergent, so we do not pursue it; but if we curtail it at the term in $\nu^{-2 n}$ ( $n$ arbitrary) we obtain a regular function $\phi(z)$ which makes the two members of (3.4) coincident up to terms of order $\nu^{-2 n+2}$. Moreover, the coincidence to this order will not be affected if, to the polynomial in $\nu^{-2}$ so determined: $z+\sum_{1}^{n} \nu^{-2 r} \phi_{r}(z)$, we add additional terms $\nu^{-2 n-2} \phi_{n+1}(z)+\cdots$ which are arbitrary apart from convergence requirements. We shall choose these terms so as to make the two members of (3.4) as nearly as possible coincident when $|z|$ is large. Thereby we shall in $\$ 4$ secure that our asymptotic formulae remain uniform up to $z=\infty$, provided $g$ satisfies suitable hypotheses.

3.1. Hypotheses. The following hypotheses are rather more general than are needed to cover Examples 2, 3 of $\$ 2$, and at the same time are sufficiently precise to lead to clear-cut results.

Hypothesis 1. There is a domain $\mathcal{D}_{z}$ in the $z$-plane, enclosing $z=0$, in which $g$ (equation (3.3)) has an expansion

$$
g\left(z, \nu^{-2}\right)=\sum_{0}^{\infty} \nu^{-2 r} g_{r}(z)
$$

the $g_{r}(z)$ are regular, and

$$
\begin{array}{ll}
\left|g_{r}(z)\right| \leqq M k^{r} & \text { for }|z| \leqq 1, \\
\left|g_{r}(z)\right| \leqq M k^{r}|z| & \text { for }|z| \geqq 1,
\end{array}
$$

$M, k$ being positive constants.

HyPoTHESIS 2. $\mathscr{D}_{z}$ is a (schlicht) star-domain relative to the centre $z=0$; it contains sectors of nonzero angles, extending to infinity, centred on the rays arg $z=0, \pm 2 \pi / 3$; and it may contain other infinite sectors of nonzero angles. Thus in Example 2, Fig. 1(iv), $\mathscr{D}_{z}$ is the whole plane, less the sector $|\arg z-\pi / 3| \leqq \epsilon,|z| \geqq(3 \pi / 2)^{2 / 3}-\epsilon(\epsilon$ arbitrary, greater than 0$)$ and the conjugate sector.

This hypothesis will become of full effect only in $\$ 4$. In the present section the essential part of it is "any point $z$ of $\mathcal{D}_{z}$ can be joined to the origin by a path whose length is less than $A|z|$, , and this would be sufficient for the most vital part of $\$ 4$ provided the exposition were suitably elaborated.

' Hypothesis 3. In any sector of $\mathcal{D}_{z}$ which extends to infinity,

$$
\begin{gathered}
g\left(z, \nu^{-2}\right)=\alpha_{\nu} z+\beta_{\nu}+O\left(z^{-1}\right), \\
\alpha_{\nu}=\sum_{0}^{\infty} \alpha_{r} \nu^{-2 r}, \quad \beta_{\nu}=\sum_{0}^{\infty} \beta_{r} \nu^{-2 r},
\end{gathered}
$$

uniformly for arg $z$ belonging to the sector and $|\nu|$ sufficiently large; the series for $\alpha_{\nu}, \beta_{\nu}$ being convergent. (There may, of course, be sectors reaching arbitrarily near to $\infty$, in which (3.7) is not satisfied.) 
As an immediate consequence we have, for any finite set of the coefficients in $(3.5)$

$$
g_{r}(z)=\alpha_{r} z+\beta_{r}+O\left(z^{-1}\right),
$$

uniformly for $\arg z$ in the sector, and this is consistent with (3.6) $)_{2}$. Hence follows $g_{r}^{\prime}(z)=\alpha_{r}+O\left(z^{-2}\right), g_{r}^{\prime \prime}(z)=O\left(z^{-3}\right)$, and so on. If (3.8) is uniform in $\theta_{1} \leqq \arg z \leqq \theta_{2}$, each derived relation is uniform in $\theta_{1}+\epsilon \leqq \arg z \leqq \theta_{2}-\epsilon$. In the sequel we shall have to perform a finite number of differentiations of polynomial combinations of the $g_{r}$, so one contraction $\epsilon$ in the sector will suffice for all; and we shall reduce the verbiage by supposing that this contraction has already been made in defining $\mathscr{D}_{z}$ : that is, (3.7) is actually valid in sectors whose angles are somewhat larger than those of $\mathcal{D}_{z}$. In each sector of Examples 2,3 the right-hand member of (3.7) is a convergent series, and no contraction of the sector is involved on differentiation.

From these hypotheses we shall deduce (\$\$3.2-3.4):

TheOREM. A transformation (3.1) may be defined which is in $\mathcal{D}_{z}$ regular, reversible, and arbitrarily near the identity for $|\nu|$ sufficiently large, whereby (3.2) is converted into a differential equation (3.9) below, in which $G$ is regular and satisfies inequalities of the form

$$
\begin{array}{ll}
|G| \leqq A_{n}|\nu|^{-2 n} & \text { for }|z| \leqq 1, \\
|G| \leqq A_{n}|\nu|^{-2 n}|z|^{-7 / 2} & \text { for }|z| \geqq 1,
\end{array}
$$

where the constant $A_{n}$ depends only on the arbitrary integer $n$.

3.2. Definition of the transformation. Consider the transform of the Airy equation (3.2) by (3.1). With a view to comparing the resulting equation with (3.3) we write it as

$$
\frac{d^{2} y}{d z^{2}}+y\left(-\nu^{2} z+g-G\right)=0
$$

where by Lemma 1

$$
G=G\left(z, \nu^{2}\right)=\nu^{2}\left(\phi \phi^{\prime 2}-z\right)+\sum_{0}^{\infty} \nu^{-2 r} g_{r}-\frac{\phi^{\prime \prime \prime}}{2 \phi^{\prime}}+\frac{3}{4}\left(\frac{\phi^{\prime \prime}}{\phi^{\prime}}\right)^{2}
$$

Substitute here $\phi=z+\sum_{1}^{\infty} \nu^{-2 r} \phi_{r}$, so that $\phi^{\prime}=1+\sum_{1}^{\infty} \nu^{-2 r} \phi_{r}^{\prime}$, and so on, and we can develop formally in powers of $\nu^{-2}$. The term in $\nu^{2}$ cancels, and equating to zero the coefficients of $\nu^{0}, \nu^{-2}, \cdots$, we get

$$
\begin{aligned}
& 2 z \phi_{1}^{\prime}+\phi_{1}=-g_{0}, \\
& 2 z \phi_{2}^{\prime}+\phi_{2}=-g_{1}-z \phi_{1}^{\prime 2}-2 \phi_{1} \phi_{1}^{\prime}+\phi_{1}^{\prime \prime \prime} / 2,
\end{aligned}
$$


which can be successively solved for $\phi_{1}, \phi_{2}, \ldots$.

The general solution for $\phi_{1}$ is

$$
z^{1 / 2} \phi_{1}=-\int_{0}^{z} 2^{-1} g_{0}(x) x^{-1 / 2} d x+C,
$$

and since $g_{0}(x)=\mathscr{P}(x)$ there is one solution $\phi_{1}$, and only one, which is regular at $z=0$, namely, the one for which $C=0$. We choose this solution; it is regular in $\mathcal{D}_{z}$ since $g_{0}$ is regular there. The right-hand member of $(3.11)_{2}$ now becomes a known function, regular in $\mathscr{D}_{z}$, so for a similar reason there is one solution $\phi_{2}$ which is regular in $\mathscr{D}_{z}$; and so on.

We suppose that the successive solution of (3.11) is terminated after $\phi_{n}$ has been found, for some chosen integer $n$. We then choose $\phi_{n+1}, \phi_{n+2}, \cdots$ so that, in the expansion of

$$
\nu^{2}\left(\phi \phi^{\prime 2}-z\right)+g,
$$

the coefficients of $\nu^{-2 n}, \nu^{-2 n-2}, \cdots$ may vanish; they are given, therefore, by equations derived from (3.11) by striking out the terms involving second or third derivatives of the $\phi_{r}$.

For formal convenience, let functions $g_{0}^{*}(z), \cdots, g_{n-1}^{*}(z)$ be defined by

$$
g_{r}^{*}(z)=g_{r}(z)+h_{r}(z) \quad(r=0, \cdots, n-1),
$$

where $h_{r}$ is the coefficient of $\nu^{-2 r}$ in the expansion of

$$
-\frac{\phi^{\prime \prime \prime}}{2 \phi^{\prime}}+\frac{3}{4}\left(\frac{\phi^{\prime \prime}}{\phi^{\prime}}\right)^{2}
$$

Thus $h_{0}=0$, and (for $r=1,2, \cdots, n-1$ ) $h_{r}$ is a polynomial in derivatives of $\phi_{1}, \cdots, \phi_{r}$, of the form

$$
h_{r}=\sum \phi_{i}^{\prime \prime \prime} \mathcal{P}\left(\phi_{j}^{\prime}\right)+\sum \phi_{i}^{\prime \prime} \phi_{j}^{\prime \prime} \mathcal{P}\left(\phi_{k}^{\prime}\right) ;
$$

and the $h_{r}, g_{r}{ }^{*}$ are determinate functions of $z$, regular in $\mathcal{D}_{z}$, since $\phi_{1}, \cdots, \phi_{n-1}$ are regular and already determined. In consequence of this definition, the equations (3.11) for $\phi_{1}, \cdots, \phi_{n}$ take the form

$g_{r}^{*}+\left[\right.$ coefficient of $\nu^{-2 r}$ in expansion of $\left.\nu^{2}\left(\phi \phi^{\prime 2}-z\right)\right]=0(r=0, \cdots, n-1)$;

so if we define

$$
g_{r}^{*}=g_{r}(r \geqq n), \quad g^{*}=\sum_{0}^{\infty} \nu^{-2 r} g_{r}^{*},
$$

the equations determining all the $\phi_{r}$ are comprised in

$$
\nu^{2}\left(\phi \phi^{\prime 2}-z\right)+g^{*}\left(z, \nu^{-2}\right)=0 .
$$

This is a differential equation for $\phi(z)$, in which the variables are separable. 
We have to pick the solution which answers to the values of $\phi_{1}, \cdots, \phi_{n}$ already determined, to demonstrate that this solution can be convergently expanded in powers of $\nu^{-2}$-which will validate the previous formal developments-and to determine the character of the solution in the infinite sectors of $\mathcal{D}_{z}$.

3.3. Properties of the $\phi_{r}(z)$ and of the series $z+\sum \nu^{-2 r} \phi_{r}(z)$. We have to discuss (i) $\phi_{1}, \cdots, \phi_{n}$, (ii) $h_{1}, \cdots, h_{n-1}$ and $g^{*}$, (iii) the solution of the differential equation (3.15); in this order.

(i) $\phi_{1}, \cdots, \phi_{n}$ have already been proved to be regular in $\mathcal{D}_{z}$. Consider their behaviour in the typical infinite sector. For any $z$ in the sector we have, by (3.8), $g_{0}(z)=\alpha_{0} z+\beta_{0}+O\left(z^{-1}\right)$, so $(3.11)_{1}$ gives

$$
\begin{aligned}
z^{1 / 2} \phi_{1}(z) & =-\frac{1}{3} \alpha_{0} z^{3 / 2}-\beta_{0} z^{1 / 2}-c_{0}+O\left(z^{-1 / 2}\right), \\
c_{0} & =\frac{1}{2} \int_{0}^{\infty}\left(g_{0}-\alpha_{0} z-\beta_{0}\right) z^{-1 / 2} d z .
\end{aligned}
$$

Hence

$$
\begin{gathered}
\phi_{1}=-\alpha_{0} z / 3-\beta_{0}-c_{0} z^{-1 / 2}+O\left(z^{-1}\right), \quad \phi_{1}^{\prime}=-\alpha_{0} / 3+c_{0} z^{-3 / 2} / 2+O\left(z^{-2}\right), \\
\phi_{1}^{\prime \prime \prime}=O\left(z^{-7 / 2}\right),
\end{gathered}
$$

and

$$
z \phi_{1}{ }^{2}+2 \phi_{1} \phi_{1}^{\prime}+\phi_{1}^{\prime \prime \prime} / 2=\alpha_{0}^{2} z / 3+2 \alpha_{0} \beta_{0} / 3+O\left(z^{-1}\right),
$$

where the term in $z^{-1 / 2}$ cancels. Since also $g_{1}=\alpha_{1} z+\beta_{1}+O\left(z^{-1}\right),(3.11)_{2}$ gives the form $\phi_{2}=a_{2} z+b_{2}+c_{2} z^{-1 / 2}+O\left(z^{-1}\right)$. An inductive proof that

$$
\phi_{r}=a_{r} z+b_{r}+c_{r} z^{-1 / 2}+O\left(z^{-1}\right) \quad(r=1,2, \cdots, n)
$$

is easily given; the essential point is that, if $\phi_{1}, \cdots, \phi_{r-1}$ have this form, then the equation (3.11), for $\phi_{r}$ has its right-hand member of the form $C z+D+O\left(z^{-1}\right)$ with no term in $z^{-1 / 2}$.

(ii) From the form (3.13) for $h_{r}$ along with the form (3.16) just demonstrated, we have

$$
h_{r}=O\left(z^{-7 / 2}\right),
$$

and thence from (3.12) and (3.8)

$$
g_{r}^{*}=g_{r}+O\left(z^{-7 / 2}\right)=\alpha_{r} z+\beta_{r}+O\left(z^{-1}\right) .
$$

It follows that, throughout $\mathcal{D}_{z},\left|g_{r}^{*}(z) / z\right|$ is bounded for $|z| \geqq 1$. Also $g_{r}^{*}(z)$, being regular in $\mathcal{D}_{z}$, is bounded for $|z| \leqq 1$. So for the finite set $g_{0}^{*}, \cdots, g_{n-1}^{*}$, we can assert inequalities of the form 


$$
\begin{array}{ll}
\left|g_{r}^{*}\right| \leqq M^{*} k^{r} & \text { for }|z| \leqq 1, \\
\left|g_{r}^{*}\right| \leqq M^{*} k^{r}|z| & \text { for }|z| \geqq 1,
\end{array}
$$

where $k$ is the same constant as in (3.6). And since $g_{r}^{*}=g_{r}$ for $r \geqq n$, and we can take $M^{*} \geqq M,(3.19)$ are valid for all $r$.

(iii) From (3.19) it follows that, in the differential equation (3.15), $g^{*}$ is an analytic function of the two variables $z, \nu^{-2}$, regular for $z$ in $\mathcal{D}_{z}$ and $\left|\nu^{2}\right|>k$. There is no difficulty in proving that there is just one solution $\phi$ which is regular in $\mathcal{D}_{z}$, and in establishing the expansibility of this solution in powers of $\nu^{-2}$. The steps are:

(a) In $\mathcal{D}_{z}, z-\nu^{-2} g^{*}$ has just one zero $z_{0}=\nu^{-2} \Phi_{1}\left(\nu^{-2}\right)$; and

$$
z-\nu^{-2} g^{*}=\left(z-z_{0}\right)\left\{1+\sum_{1}^{\infty} \nu^{-2 r} f_{r}(z)\right\}, \quad \text { with }\left|f_{r}\right| \leqq M_{1} k_{1}^{r} \text {. }
$$

(b) The only solution $\phi$ which is regular in $\mathscr{D}_{z}$ is given by

$$
\frac{2}{3} \phi^{3 / 2}=\int_{z_{0}}^{z}\left\{x-\nu^{-2} g^{*}\left(x, \nu^{-2}\right)\right\}^{1 / 2} d x .
$$

(c) In $\mathscr{D}_{z}, \phi$ is expansible in the form

$$
\phi=\left(z-z_{0}\right)\left\{1+\sum_{1}^{\infty} \nu^{-2 r} f_{r}^{*}(z)\right\}
$$

with $\left|f_{r}^{*}(z)\right| \leqq M_{2} k_{2}^{r}$

in deducing these inequalities from (3.20) we appeal to hypothesis 2 of $\$ 3.1$.

(d) By substituting from (3.20), (3.21) in (3.15) we get a development

$$
\phi^{\prime}=1+\sum_{1}^{\infty} \nu^{-2 r} \phi_{r}^{* \prime}(z), \quad \text { with }\left|\phi_{r}^{* \prime}(z)\right| \leqq M_{3} k_{3}^{r} \text {. }
$$

An integral of this,

$$
\phi=z+\sum_{1}^{\infty} \nu^{-2 r} \phi_{r}^{*}(z)
$$

must agree with the series obtained from (3.21) by substituting for $z_{0}$ its value $\nu^{-2} \Phi_{1}\left(\nu^{-2}\right)$ and rearranging; and

$$
\begin{array}{ll}
\left|\phi_{r}^{*}(z)\right| \leqq M_{3}^{*} k_{3}^{r} & \text { for }|z| \leqq 1, \\
\left|\phi_{r}^{*}(z)\right| \leqq M_{3}^{*} k_{3}^{r}|z| . & \text { for }|z| \geqq 1 .
\end{array}
$$

(e) The series for $\phi^{\prime \prime}, \phi^{\prime \prime \prime}$ obtained by differentiating (3.22) converge uniformly in $\mathcal{D}_{z}$, apart from a strip round its boundary (Weierstrass), but it is not necessary to remove such a strip since the series can be obtained from derivatives of the differential equation (3.15). 
Substituting this solution $\phi$ in (3.10), $G$ becomes an analytic function of $z$ and $\nu$, regular for $z$ in $\mathcal{D}_{z}$ and $|\nu|$ sufficiently large. The expansion leading to the equations (3.11) is justified, and the $\phi_{1}, \cdots, \phi_{n}$ thence determined must agree with $\phi_{1}^{*}, \cdots, \phi_{n}^{*}$ in (3.22) since the solutions which are regular in $\mathcal{D}_{z}$ are unique.

In the typical infinite sector of $\mathcal{D}_{z}$ we have, from (3.14), (3.18), and (3.7),

$$
z-\nu^{-2} g^{*}=z-\nu^{-2} g+\sum_{1}^{n-1} \nu^{-2 r-2}\left(g_{r}-g_{r}^{*}\right)=z-\nu^{-2}\left\{\alpha_{\nu} z+\beta_{\nu}+O\left(z^{-1}\right)\right\},
$$

uniformly as to $\nu$ and arg $z$. Substituting in (3.15) we obtain, uniformly,

$$
\begin{aligned}
2 \phi^{3 / 2} / 3 & =\left(2\left(1-\nu^{-2} \alpha_{\nu}\right)^{1 / 2} / 3\right) z^{3 / 2}+\nu^{-2} \beta_{\nu}\left(1-\nu^{-2} \alpha_{\nu}\right)^{-1 / 2} z^{1 / 2} \\
& +\nu^{-2} c_{\nu}+O\left(\nu^{-2} z^{-1 / 2}\right)
\end{aligned}
$$

where

$(3.24)_{2} \nu^{-2} c_{\nu}=\lim _{z \rightarrow \infty}\left\{2 \phi^{3 / 2} / 3-\left(2\left(1-\nu^{-2} \alpha_{\nu}\right)^{1 / 2} / 3\right) z^{3 / 2}-\nu^{-2} \beta_{\nu}\left(1-\nu^{-2} \alpha_{\nu}\right)^{-1 / 2} z^{1 / 2}\right\}$.

Also

$$
\lim _{z \rightarrow \infty} \frac{\phi \phi^{\prime 2}}{z}=1-\nu^{-2} \alpha_{\nu}
$$

3.4. Inequalities for $G$. It has been proved that, with $\phi(z)$ determined as in $\$ 3.2,(3.10)$ gives $G$ regular for $z$ in $\mathcal{D}_{z}$ and $|\nu|$ sufficiently large; and when we expand $G$ in powers of $\nu^{-2}$, the terms in $\nu^{0}, \cdots, \nu^{-2 n+2}$ vanish on account of the choice of $\phi_{1}, \cdots, \phi_{n}$. Hence $\nu^{2 n} G$ is regular.

Now if we substitute (3.15) in (3.10) and take note of (3.14), (3.12) we get

$$
\begin{aligned}
G & =-\frac{\phi^{\prime \prime \prime}}{2 \phi^{\prime}}+\frac{3}{4}\left(\frac{\phi^{\prime \prime}}{\phi^{\prime}}\right)^{2}+\sum_{1}^{\infty} \nu^{-2 r}\left(g_{r}-g_{r}^{*}\right) \\
& =-\frac{\phi^{\prime \prime \prime}}{2 \phi^{\prime}}+\frac{3}{4}\left(\frac{\phi^{\prime \prime}}{\phi^{\prime}}\right)^{2}-\sum_{1}^{n-1} \nu^{-2 r} h_{r} .
\end{aligned}
$$

In the typical infinite sector of $\mathcal{D}_{z},(3.17)$ gives $h_{r}=O\left(z^{-7 / 2}\right)$, while (3.24) gives $\phi^{\prime}=1+O\left(\nu^{-2}\right), \phi^{\prime \prime}=O\left(\nu^{-2} z^{-5 / 2}\right), \phi^{\prime \prime \prime}=O\left(\nu^{-2} z^{-7 / 2}\right)$. Hence $G=O\left(\nu^{-2} z^{-7 / 2}\right)$ uniformly as to $\nu$ and $\arg z$.

Hence for $|\nu| \geqq \nu_{0}$, say, and $z$ in $\mathcal{D}_{z}$, there are inequalities of the form

$$
|G| \leqq A_{n} \quad \text { for } \quad|z| \leqq 1, \quad|G| \leqq A_{n}|z|^{-7 / 2} \text { for }|z| \geqq 1 \text {; }
$$

and since $\nu^{2 n} G$ is regular, the maximum modulus theorem gives

$$
\begin{array}{ll}
|G| \leqq A_{n} \nu_{0}^{2 n}|\nu|^{-2 n} & \text { for }|z| \leqq 1, \\
|G| \leqq A_{n} \nu_{0}^{2 n}|\nu|^{-2 n}|z|^{-7 / 2} & \text { for }|z| \geqq 1 .
\end{array}
$$


This completes the proof of the theorem stated at the end of $\$ 3.1$.

3.5. On the value of $c_{\nu}$, equation (3.24), in a special case. Consider the case in which (i) the function $g\left(z, \nu^{-2}\right)$ in (3.3) is real when $z, \nu^{2}$ are real, and (ii) one of the infinite sectors of $\mathcal{D}_{z}$ encloses the ray arg $z=\pi$. From (i), when $z, \nu^{2}$ are real all the $\phi_{r}$ are real, so $\phi(z)$ is real; and on the ray $\arg z=\pi, \phi(z)$ has the principal part $z$ and so is real negative. It follows that in (3.24)

$c_{\nu}$ must be pure-imaginary when $\nu^{2}$ is real;

more precisely, if $\phi^{3 / 2}, z^{1 / 2}$ are to stand for principal values, there are two forms of (3.24) in the sector:

$2 \phi^{3 / 2} / 3=\left(2\left(1-\nu^{-2} \alpha_{\nu}\right)^{1 / 2} / 3\right) z^{3 / 2}+\nu^{-2} \beta_{\nu}\left(1-\nu^{-2} \alpha_{\nu}\right)^{-1 / 2} z^{1 / 2} \pm i d_{\nu} \nu^{-2}+O\left(\nu^{-2} z^{-1 / 2}\right)$,

one of which must be used for $\arg z \leqq \pi$ and the other for $\arg z \geqq-\pi$; and $d_{\nu}$ is real when $\nu^{2}$ is real.

4. Airy-function approximations to the solutions of (3.3). For this part of the investigation we start from the differential equation (3.3) for the "unknown" functions and the almost coincident equation (3.9) into which we have transformed the Airy equation (3.2). For comparing the solutions of these it is convenient to work with a variable in which the approximationfunctions are as simple as possible, and moreover to work with equations in canonic form B. We therefore reconvert (3.9) into (3.2) by reversing the transformation $\left(^{5}\right) \zeta=\phi(z)$, and by then putting $\zeta=(3 v / 2)^{2 / 3}, \eta=w \zeta^{-1 / 4}$ we get

$$
\frac{d^{2} w}{d v^{2}}+w\left(-\nu^{2}+\frac{5}{36 v^{2}}\right)=0 .
$$

In applying the same transformations to (3.3) we use for the new dependent variable a symbol $\bar{\omega}$ distinct from $w$. The transformation is

$$
\begin{array}{ll}
\zeta=(3 v / 2)^{2 / 3}, & z=\phi^{-1}(\zeta)=\phi^{-1}\left[(3 v / 2)^{2 / 3}\right]=\psi(v), \\
y=\bar{\omega}\left(\psi^{\prime}(v)\right)^{1 / 2}, & \psi^{\prime}=\frac{1}{\zeta^{1 / 2} \phi^{\prime}}=\left(\frac{2}{3 v}\right)^{1 / 3} \cdot \frac{1}{\phi^{\prime}},
\end{array}
$$

and by Lemma 1 it converts (3.3) into

$$
\frac{d^{2} \bar{\omega}}{d v^{2}}+\bar{\omega}\left[-\nu^{2} z \psi^{\prime 2}+g \psi^{\prime 2}+\frac{\psi^{\prime \prime \prime}}{2 \psi^{\prime}}-\frac{3}{4}\left(\frac{\psi^{\prime \prime}}{\psi^{\prime}}\right)^{2}\right]=0 .
$$

Now (3.9) is obtained from (3.3) on replacing $g$ by $g-G$, so the same replacement in (4.3) must yield (4.1). Hence (4.3) must be

$$
\frac{d^{2} \bar{\omega}}{d v^{2}}+\bar{\omega}\left[-\nu^{2}+\frac{5}{36 v^{2}}+F\left(v, \nu^{-2}\right)\right]=0,
$$

(5) This transformation depends upon $n$, but it seems unnecessary to show this in the notation. 
where

$$
F=G \psi^{\prime 2}=\frac{G}{\phi^{\prime 2}}\left(\frac{2}{3 v}\right)^{2 / 3} .
$$

When $|\nu|$ is large, the domain $\mathcal{D}_{z}$ is in one-one correspondence with an almost coincident domain $\mathcal{D}_{\zeta, \nu}$, and this, via $\zeta=(3 v / 2)^{2 / 3}$, is in $1: 2$ correspondence with a part $\mathcal{D}_{v, \nu}$ of a 3 -sheeted Riemann surface having the branch point $v=0$ in its interior. Thus in Example 2 of $\S 2, \mathcal{D}_{v, v}$ is the whole of the Riemann surface of $v^{1 / 3}$, except for arbitrary sectorial neighbourhoods of four cuts one of which is $C^{\prime} G$ in Fig. 1 (iii). The notation $\mathcal{D}_{v, v}$ exhibits the dependence of the boundaries upon $\nu$, but for almost all purposes their small variation with $\nu$ is unimportant; since $\mathcal{D}_{z}$ is a star (centre $z=0$ ), $\mathscr{D}_{v, v}$ is almost a star (centre $v=0$ ), and we can make it strictly a star by paring away a thin layer (depending on $\nu$ ) from its boundary.

The relevant properties of $F$ in (4.4) are that it is regular in $\mathcal{D}_{v, v}$, except at $v=0$, and that

$$
\begin{array}{ll}
\left|F\left(v, \nu^{-2}\right)\right| \leqq A_{n}|\nu|^{-2 n}|v|^{-2 / 3} & \text { for }|v| \leqq 1, \\
\left|F\left(v, \nu^{-2}\right)\right| \leqq A_{n}|\nu|^{-2 n}|v|^{-3} & \text { for }|v| \geqq 1 ;
\end{array}
$$

these follow from (4.5) and (3.26), if we bear in mind that $\zeta=(3 v / 2)^{2 / 3}$ and $\phi^{\prime}=1+O\left(\nu^{-2}\right)$, uniformly.

The comparison of solutions of (4.1) and (4.4) rests upon the following simple lemma:

LEMMA 2. Let $w_{1}(v), w_{2}(v)$ be linearly independent solutions of (4.1), let: $w(v)$ be any solution, and let $\bar{\omega}(v)$ be a function satisfying

$$
\bar{\omega}(v)=w(v)+\frac{1}{\Delta} \int_{v_{0}}^{v}\left\{w_{2}(v) w_{1}(t)-w_{1}(v) w_{2}(t)\right\} F(t) \bar{\omega}(t) d t,
$$

where

$$
\Delta=w_{1}(v) w_{2}^{\prime}(v)-w_{2}(v) w_{1}^{\prime}(v) \neq 0 ;
$$

the integral may be improper at $v_{0}$ provided that it converges there. Then $\bar{\omega}(v)$ is a solution of (4.4).

Starting with given $w_{1}, w_{2}, w$ we prove in $\$ 4.2$ the existence of a solution $\bar{\omega}$ of the integral equation (4.7), and deduce the asymptotic formula $\bar{\omega} / w$ $=1+O\left(\nu^{-2 n-1}\right)$, provided $v$ is suitably restricted. The transformation (4.2) then (\$4.3) gives a similar asymptotic formula for a certain solution $y$ of (3.3). To deduce asymptotic formulae for standard solutions of (3.3) we must express $y$ in terms of such solutions ( $\$ 4.4$ ), and it is because this is most easily done by choosing in (4.7) $v_{0}=+\infty$ or $\infty e^{ \pm \pi i}$ that we have taken trouble in $\S 3$ to make $F$ integrable to infinity, according to $(4.6)_{2}$. The final step $(\$ 4.5)$ is to find the largest domains of validity of the asymptotic formulae; the ones 
first found are unnecessarily restricted.

In $\$ \$ 4.2-4.5$ we restrict $\nu$ to be real positive. In $\$ 4.6$ the restriction is relaxed.

4.1. Properties of the approximation-function $L(x)$. The equation (4.1) is a well known transform of Bessel's equation of order $1 / 3$. We shall use the following facts regarding its solutions:

(i) The general solution is

$$
w=C L(\nu v)+D L\left(\nu v e^{ \pm \pi i}\right),
$$

where

$$
L(x)=x^{1 / 2} K_{1 / 3}(x)=x^{1 / 6} \mathcal{P}_{1}\left(x^{2}\right)+x^{5 / 6} \mathcal{P}_{2}\left(x^{2}\right),
$$

the power series $\Phi_{1}, \Phi_{2}$ being everywhere convergent. The multiple-valued $L(x)$ is made determinate on a 6 -sheeted Riemann surface by taking arg $x^{1 / 6}$, $\arg x^{5 / 6}$ to vanish when $\arg x=0$.

(ii) $L(x)$ and $L\left(x e^{-\pi i}\right)$ are linearly independent, their Wronskian being

$$
L(x) \frac{d}{d x} L\left(x e^{-\pi i}\right)-L\left(x e^{-\pi i}\right) \frac{d}{d x} L(x)=\pi .
$$

(iii) For $|x| \sim \infty$,

$$
L(x) \sim(\pi / 2)^{1 / 2} \cdot e^{-x},
$$

uniformly for $|\arg x| \leqq 3 \pi / 2-\epsilon$.

(iv) $L(x)$ has strings of zeros on the rays arg $x= \pm 3 \pi / 2$, the smallest being at about $|x|=2.3$; for $|\arg x|<3 \pi / 2$ it is nonzero.

From these, along with the corresponding properties of $L\left(x e^{-x i}\right)$, we easily prove that there are inequalities of the form

$$
|L(x)| \leqq A_{1}\left|e^{-x}\right|, \quad\left|L\left(x e^{-\pi i}\right)\right| \leqq A_{1}\left|e^{x}\right|, \quad\left|\frac{L\left(x e^{-\pi i}\right)}{L(x)}\right| \leqq A_{2}\left|e^{2 x}\right|,
$$

valid for $-\pi / 2+\epsilon \leqq \arg x \leqq 3 \pi / 2-\epsilon$, without restriction upon $|x|$.

4.2. Solution of the integral equation (4.7). Let us take $w(v)=w_{1}(v)=L(\nu v)$, $w_{2}(v)=L\left(\nu v e^{-\pi i}\right)$, and $v_{0}=+\infty$. Inserting in (4.7) the value of the Wronskian, $\Delta=\nu \pi$, from (4.9), we write the integral equation in the form

$$
\frac{\bar{\omega}(v)}{L(\nu v)}=1+\frac{1}{\nu \pi} \int_{v}^{+\infty}\left\{L(\nu t) L\left(\nu t e^{-\pi i}\right)-L^{2}(\nu t) \frac{L\left(\nu v e^{-\pi i}\right)}{L(\nu v)}\right\} \frac{F(t) \bar{\omega}(t)}{L(\nu t)} d t .
$$

Taking $\nu$ to be real positive, we can use the inequalities (4.11) with $x=\nu t$ or $\nu v$, provided we restrict $v$ and all points $t$ on the path of integration thence to $+\infty$ by the condition

$$
-\pi / 2+\epsilon \leqq \arg v, \arg t \leqq 3 \pi / 2-\epsilon .
$$


Then in the integrand of (4.12)

$$
\left|L(\nu t) L\left(\nu t e^{-\pi i}\right)-L^{2}(\nu t) \frac{L\left(\nu v e^{-\pi i}\right)}{L(\nu v)}\right| \leqq A_{1}^{2}+A_{1}^{2} A_{2}\left|e^{-2 \nu(t-v)}\right|,
$$

and to make this less than $A_{1}^{2}+A_{1}^{2} A_{2}$ we impose the further restriction

$$
-\pi / 2 \leqq \arg (t-v) \leqq \pi / 2,
$$

for all points $t$ of the path from $v$ to $+\infty$. It is clear that this will be satisfied provided the slope of the path (away from $v$ ) is everywhere between $\pm \pi / 2$, inclusive.

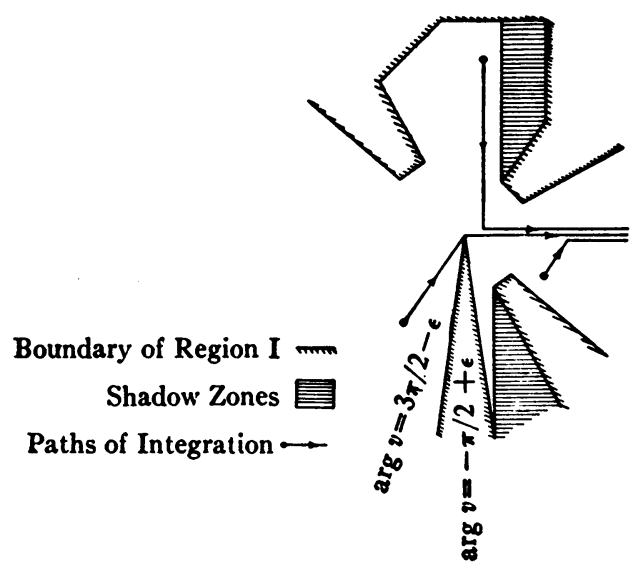

FIG. 3

Region I, showing shadow-zones and typical paths of integration.

We call Region I the part of $\mathcal{D}_{v, \nu}$ for which these two restrictions are satisfied. Since $\mathscr{D}_{v, v}$ can be taken as a star (centre $v=0$ ), the condition (4.15) is of no effect where $\operatorname{Re} v \leqq 0$, but we must exclude any zone in the quadrant $0<\arg v<\pi / 2$ which, on account of an intervening boundary of $\mathcal{D}_{v, v}$, cannot be "seen" from $v=-i \infty$; and similarly for the conjugate quadrant. These excluded zones are called shadow-zones; see Fig. 3, which shows also suitable paths of integration.

The integral equation (4.12) is now solved by iteration. Write $\bar{\omega}(v) / L(\nu v)$ $=f(v)$, and define

$$
\begin{gathered}
f_{-1}(v)=0, \quad f_{0}(v)=1 \\
f_{r+1}(v)=1+\frac{1}{\nu \pi} \int_{v}^{+\infty}\left\{L(\nu t) L\left(\nu t e^{-\pi i}\right)-L^{2}(\nu t) \frac{L\left(\nu v e^{-\pi i}\right)}{L(\nu v)}\right\} F(t) f_{r}(t) d t .
\end{gathered}
$$


Then for $r=0,1,2, \cdots$

$$
\begin{aligned}
f_{r+1}(v)- & f_{r}(v) \\
& =\frac{1}{\nu \pi} \int_{v}^{+\infty}\left\{L(\nu t) L\left(\nu t e^{-\pi i}\right)-L^{2}(\nu t) \frac{L\left(\nu v e^{-\pi i}\right)}{L(\nu v)}\right\} F(t)\left\{f_{r}(t)-f_{r-1}(t)\right\} d t,
\end{aligned}
$$

and from (4.14), (4.15)

$$
\left|f_{r+1}(v)-f_{r}(v)\right| \leqq(\nu \pi)^{-1} A_{1}^{2}\left(1+A_{2}\right) \cdot\left|f_{r}(t)-f_{r-1}(t)\right|_{\max } \cdot \int_{v}^{+\infty}|F(t) d t| .
$$

Here, by (4.6), the integral is less than an absolute multiple of $|\nu|^{-2 n}$, for any $v$ in Region I, so $\left|f_{r+1}(v)-f_{r}(v)\right| \leqq A_{n} \nu^{-2 n-1}\left|f_{r}(t)-f_{r-1}(t)\right|_{\max }$.

It follows that $f_{r+1}(v)-f_{r}(v) \rightarrow 0$ as $r \rightarrow \infty$, uniformly for $v$ in Region I, provided $\nu>\left(A_{n}+\epsilon\right)^{1 /(2 n+1)}$; and $f_{r}(v)$ tends uniformly to a limit $f(v)$, and $f(v)$ is bounded in Region I. By letting $r \rightarrow \infty$ in (4.16) we prove that $\bar{\omega}(v)$, $=f(v) L(\nu v)=\bar{\omega}_{1}(v)$, say, is a solution of (4.12); and by inserting the upper bound of $\left|\bar{\omega}_{1}(t) / L(\nu t)\right|$ on the right of (4.12) we prove (i) that

$$
\frac{\bar{\omega}_{1}(v)}{L(\nu v)}=1+O\left(\nu^{-2 n-1}\right) \text {, }
$$

uniformly in Region I; and, (ii), using (4.6) 2 , that

$$
\frac{\bar{\omega}_{1}(v)}{L(\nu v)}=1+O\left(\nu^{-2 n-1} v^{-2}\right) \quad \text { for } v \sim+\infty .
$$

From (4.8) follows the identity

$$
L\left(x e^{-\pi i}\right)=L\left(x e^{\pi i}\right)-i L(x),
$$

and by use of this (4.12) is converted into the conjugate equation, which is therefore satisfied by $\bar{\omega}_{1}(v)$. But for the conjugate equation the condition permitting iterative solution is $-3 \pi / 2+\epsilon \leqq \arg v \leqq \pi / 2-\epsilon$, in place of (4.13). Hence we can redefine Region I with $|\arg v| \leqq 3 \pi / 2-\epsilon$ in place of (4.13), and (4.17), (4.18) are valid in the enlarged region.

In the same way, taking $w=w_{1}=L\left(\nu v e^{-\pi i}\right), w_{2}=L(\nu v)$ or $L\left(\nu v e^{-2 \pi i}\right)$, and $v_{0}=\infty e^{x i}$ in (4.7), we find a solution $\bar{\omega}_{2}(v)$ of (4.4) for which, uniformly in a Region II of $\mathscr{D}_{v, \nu}$,

$$
\frac{\bar{\omega}_{2}(v)}{L\left(\nu v e^{-\pi i}\right)}=1+O\left(\nu^{-2 n-1}\right) .
$$

And similarly there is a solution $\bar{\omega}_{3}(v)$ for which, in a Region III,

$$
\frac{\bar{\omega}_{3}(v)}{L\left(\nu v e^{\pi i}\right)}=1+O\left(\nu^{-2 n-1}\right) \text {. }
$$


To these correspond formulae like (4.18) for $v \sim \infty e^{\pi i}, \infty e^{-\pi i}$ respectively. For Region II the restriction is $-\pi / 2+\epsilon \leqq \arg v \leqq 5 \pi / 2+\epsilon$, with the possible exclusion of shadow-zones in the half-plane $\pi / 2<\arg v<3 \pi / 2$; and Region III is its conjugate.

4.3. Airy-function formulae. By applying the transformation (4.2) we convert (4.17), and so on, into asymptotic formulae for solutions $y_{1}(z)$, and so on, of the differential equation (3.3) which is our primary concern; and to match this transformation we express the approximation-functions $L(\nu v)$, and so on, in terms of Airy functions. If

$$
\begin{gathered}
v=2 \zeta^{3 / 2} / 3, \quad \omega=\exp (2 \pi i / 3), \\
L(\nu v)=2^{1 / 2} \pi \nu^{1 / 6} \zeta^{1 / 4} \cdot \operatorname{Ai}\left(\nu^{2 / 3} \zeta\right), \\
L\left(\nu v e^{\pi i}\right)=2^{1 / 2} \pi \nu^{1 / 6} \zeta^{1 / 4} \cdot(-i \omega) \mathrm{Ai}\left(\nu^{2 / 3} \omega \zeta\right), \\
L\left(\nu v e^{-\pi i}\right)=2^{1 / 2} \pi \nu^{1 / 6} \zeta^{1 / 4} \cdot i \omega^{2} \mathrm{Ai}\left(\nu^{2 / 3} \omega^{2} \zeta\right),
\end{gathered}
$$

and the transformation gives

$$
\begin{aligned}
\frac{y_{1}(z)\left(\phi^{\prime}(z) / 2\right)^{1 / 2}}{\pi \nu^{1 / 6} \mathrm{Ai}\left(\nu^{2 / 3} \zeta\right)} & =1+O\left(\nu^{-2 n-1}\right), \\
\frac{y_{2}(z)\left(\phi^{\prime}(z) / 2\right)^{1 / 2}}{i \omega^{2} \pi \nu^{1 / 6} \mathrm{Ai}\left(\nu^{2 / 3} \omega^{2} \zeta\right)} & =1+O\left(\nu^{-2 n-1}\right), \\
\frac{y_{3}(z)\left(\phi^{\prime}(z) / 2\right)^{1 / 2}}{(-i \omega) \pi \nu^{1 / 6} \mathrm{Ai}\left(\nu^{2 / 3} \omega \zeta\right)} & =1+O\left(\nu^{-2 n-1}\right) .
\end{aligned}
$$

(4.24) is valid in a Region I of $\mathcal{D}_{\zeta, \nu}$ (or in an almost coincident Region I of $\left.\mathcal{D}_{z}\right)$, defined by $|\arg \zeta| \leqq \pi-2 \epsilon / 3$, with shadow-zones excluded corresponding to those excluded from $\mathcal{D}_{v, \nu}$; the "free" boundaries of these zones in the $\zeta$ plane, that is, the ones corresponding to the boundaries $\operatorname{Re} v=$ const., are of course curved. Thus, apart from shadow-zones, (4.24) is valid in the whole of $\mathcal{D}_{\zeta, \nu}$ except a narrow sector containing the ray arg $\zeta= \pm \pi$ on which lie the zeros of $\operatorname{Ai}\left(\nu^{2 / 3} \zeta\right)$; and for (4.25) similarly the excluded regions are sectors centred on $\arg \zeta=\mp \pi / 3$, respectively.

4.4. Identification of the solutions. Since $y_{1}(z)$ is a solution of (3.3) it is a linear combination of "standard" solutions of this equation. Now when a differential equation has singular points, its standard solutions are usually defined in relation thereto. For $\zeta \rightarrow+\infty, \mathrm{Ai}\left(\nu^{2 / 3} \zeta\right) \rightarrow 0$ and $\mathrm{Ai}\left(\nu^{2 / 3} \omega^{2} \zeta\right) \rightarrow \infty$, so since (4.24), (4.25) are uniformly valid for $\zeta \sim+\infty$, we have $y_{1}(z) \rightarrow 0, y_{2}(z) \rightarrow \infty$ as $z \rightarrow+\infty$. Hence $z=+\infty$ must be a singular point of the differential equation (3.3), and there will be just one standard solution, $Y_{v 1}(z)$, say, which is zero there. Since $y_{1}(z) \rightarrow 0$ it must be a multiple of $Y_{\nu 1}(z)$, say $y_{1}(z)=P_{\nu 1} Y_{\nu 1}(z)$, where $P_{\nu 1} \neq 0$, and (4.24) becomes

$$
\frac{P_{\nu 1} Y_{\nu 1}(z)\left(\phi^{\prime}(z) / 2\right)^{1 / 2}}{\pi \nu^{1 / 6} \mathrm{Ai}\left(\nu^{2 / 3} \zeta\right)}=1+O\left(\nu^{-2 n-1}\right) .
$$


The value of $P_{\nu 1}$ is to be found by letting $z, \zeta \rightarrow+\infty$ in (4.26), $\nu$ remaining fixed. From (4.18) we see that the right-hand member then tends to 1 . $R e$ garding the limit on the left, we have from (4.10), (4.23),

$$
\pi \nu^{1 / 6} \mathrm{Ai}\left(\nu^{2 / 3} \zeta\right)=\left(\pi^{1 / 2} / 2\right) \zeta^{-1 / 4} \exp \left(-2 \nu \zeta^{3 / 2} / 3\right)\left\{1+O\left(\nu^{-1} \zeta^{-3 / 2}\right)\right\},
$$

and here $\zeta=\phi(z)$. Substituting the limiting forms of $\phi^{3 / 2}(z)$ from $(3.24)_{1}$ and of $\phi^{\prime}(z)$ from (3.25), we obtain therefore

$$
\begin{aligned}
P_{\nu 1}=\lim _{2 \rightarrow+\infty}(\pi / 2)^{1 / 2} 2\left(1-\nu^{-2} \alpha_{\nu}\right)^{1 / 2} z^{3 / 2} / 3 \\
\cdot \frac{\exp \left[-\nu\left\{\nu^{-2} \beta_{\nu}\left(1-\nu^{-2} \alpha_{\nu}\right)^{-1 / 2} z^{1 / 2}+\nu^{-2} c_{\nu}\right\}\right]}{\left(1-\nu^{-2} \alpha_{\nu}\right)^{1 / 4} z^{1 / 4} Y_{\nu 1}(z)} .
\end{aligned}
$$

The constants $\alpha_{\nu}, \beta_{\nu}$ are determined solely from the behaviour of $g\left(z, \nu^{-2}\right)$ for $z \sim+\infty$, according to (3.7), but $c_{\nu}$ depends on the transformation $\zeta=\phi(z)$, being defined by $(3.24)_{2}$.

Similarly $y_{2}, y_{3}$ are multiples of standard solutions $Y_{\nu 2}, Y_{\nu 3}$ of (3.3), defined in relation to singular points at $z=\omega \infty, \omega^{2} \infty$ respectively, and (4.25) become

$$
\begin{aligned}
& \frac{P_{\nu 2} Y_{\nu 2}(z)\left(\phi^{\prime}(z) / 2\right)^{1 / 2}}{i \omega^{2} \pi \nu^{1 / 6} \mathrm{Ai}\left(\nu^{2 / 3} \omega^{2} \zeta\right)}=1+O\left(\nu^{-2 n-1}\right), \\
& \frac{P_{\nu 3} Y_{\nu 3}(z)\left(\phi^{\prime}(z) / 2\right)^{1 / 2}}{(-i \omega) \pi \nu^{1 / 6} \mathrm{Ai}\left(\nu^{2 / 3} \omega \zeta\right)}=1+O\left(\nu^{-2 n-1}\right),
\end{aligned}
$$

where $P_{\nu 2}, P_{\nu 3}$ are given by formulae like (4.27).

4.5. Extension of the regions of validity of (4.26), (4.28). We shall prove that (4.26) is valid throughout the sector $|\arg (-\zeta)| \leqq 2 \epsilon / 3$, hitherto omitted from Region I, except in specified neighbourhoods of the zeros of $\mathrm{Ai}\left(\nu^{2 / 3} \zeta\right)$ where a trivial modification is required.

We observe that, in $\mathcal{D}_{v, \nu}$, Regions I, II, III all include a sector centred on the ray $\arg v=0$, so (4.26), (4.28) are all valid in a sector $S$ centred on $\arg \zeta$ $=0$. Now there is a linear relation

$$
Q_{\nu 1} Y_{\nu 1}(z)+Q_{\nu 2} Y_{\nu 2}(z)+Q_{\nu 3} Y_{\nu 3}(z)=0,
$$

where $Q_{\nu 1}: Q_{\nu 2}: Q_{\nu 3}$ are determinate solely from the definitions of the standard solutions $Y_{v r}$. Hence, denoting by the generic symbol $O$ a function which is uniformly $O\left(\nu^{-2 n-1}\right)$ in $S$, we have

$$
\begin{aligned}
\frac{Q_{\nu 1}}{P_{\nu 1}} \mathrm{Ai}\left(\nu^{2 / 3} \zeta\right)(1+O)+\frac{i \omega^{2} Q_{\nu 2}}{P_{\nu 2}} \mathrm{Ai}\left(\nu^{2 / 3} \omega^{2} \zeta\right)(1+O) \\
\quad-\frac{i \omega Q_{\nu 3}}{P_{\nu 3}} \mathrm{Ai}\left(\nu^{2 / 3} \omega \zeta\right)(1+O)=0
\end{aligned}
$$

Also 


$$
\mathrm{Ai}\left(\nu^{2 / 3} \zeta\right)+\omega^{2} \mathrm{Ai}\left(\nu^{2 / 3} \omega^{2} \zeta\right)+\omega \mathrm{Ai}\left(\nu^{2 / 3} \omega \zeta\right)=0 .
$$

Eliminating $\left({ }^{6}\right) \mathrm{Ai}\left(\nu^{2 / 3} \omega^{2} \zeta\right)$ and letting $\zeta \rightarrow \infty$ in $\delta$, with $\nu$ fixed, we obtain (since $\left.\mathrm{Ai}\left(\nu^{2 / 3} \zeta\right) / \mathrm{Ai}\left(\nu^{2 / 3} \omega^{2} \zeta\right) \rightarrow 0\right), Q_{\nu 2} / P_{\nu 2}=-\left(Q_{\nu 3} / P_{\nu 3}\right)(1+O)$. Then let $\zeta \rightarrow 0$ in $\mathcal{S}$, so that the Airy functions all approach the same nonzero limit, and we obtain

$$
\frac{Q_{\nu 1}}{P_{\nu 1}}=\frac{i Q_{\nu 2}}{P_{\nu 2}}(1+O)=-\frac{i Q_{\nu 3}}{P_{\nu 3}}(1+O) .
$$

As a corollary, the $Q_{v r}$ are, like the $P_{v r}$, nonzero.

Now (4.29), (4.32) give, at all points,

$$
P_{\nu 1} Y_{\nu 1}(z)=i P_{\nu 2} Y_{\nu 2}(z)(1+O)-i P_{\nu 3} Y_{\nu 3}(z)(1+O),
$$

and since (4.28) are both valid in the sector $|\arg (-\zeta)| \leqq 2 \epsilon / 3$ we have here

$$
\begin{aligned}
P_{\nu 1} Y_{p 1}(z)\left(\phi^{\prime}(z) / 2\right)^{1 / 2}= & -\pi \nu^{1 / 6} \omega^{2} \mathrm{Ai}\left(\nu^{2 / 3} \omega^{2} \zeta\right)(1+O) \\
& -\pi \nu^{1 / 6} \omega \mathrm{Ai}\left(\nu^{2 / 3} \omega \zeta\right)(1+O) ;
\end{aligned}
$$

and thence from (4.31)

$$
\begin{aligned}
P_{\nu 1} Y_{\nu 1}(z)\left(\phi^{\prime}(z) / 2\right)^{1 / 2} & =\pi \nu^{1 / 6}\left\{\mathrm{Ai}\left(\nu^{2 / 3} \zeta\right)(1+O)+\mathrm{Ai}\left(\nu^{2 / 3} \omega \zeta\right) \cdot O\right\}, \\
& =\pi \nu^{1 / 6}\left\{\mathrm{Ai}\left(\nu^{2 / 3} \zeta\right)(1+O)+\mathrm{Ai}\left(\nu^{2 / 3} \omega^{2} \zeta\right) \cdot O\right\} .
\end{aligned}
$$

Surrounding each zero $-\zeta_{r}$ of $\mathrm{Ai}\left(\nu^{2 / 3} \zeta\right)$ there is a neighbourhood, of radius $C \nu^{-1} \zeta_{r}^{-1 / 2}$ with $C=1$, say, in which all three Airy functions are less in absolute value than $\nu^{-1 / 6} \zeta_{r}^{-1 / 4}$; these neighbourhoods do not overlap and enclose no zeros of $\mathrm{Ai}^{\prime}\left(\nu^{2 / 3} \zeta\right)\left({ }^{7}\right)$; and outside them, $\mathrm{Ai}\left(\nu^{2 / 3} \omega \zeta\right) / \mathrm{Ai}\left(\nu^{2 / 3} \zeta\right)$ is bounded on one side of the ray $\arg \zeta=\pi$, while $\mathrm{Ai}\left(\nu^{2 / 3} \omega^{2} \zeta\right) / \mathrm{Ai}\left(\nu^{2 / 3} \zeta\right)$ is bounded on the other side $\left.{ }^{8}\right)$. Hence (4.26) is valid outside the said neighbourhoods, while within them we have

$$
P_{\nu 1} Y_{\nu 1}(z)\left(\phi^{\prime}(z) / 2\right)^{1 / 2}=\pi \nu^{1 / 6} \mathrm{Ai}\left(\nu^{2 / 3} \zeta\right)+O\left(\nu^{-2 n-1} \zeta^{-1 / 4}\right) .
$$

Similarly, (4.28) are valid throughout $\mathcal{D}_{\zeta, \nu}$, except in the relevant shadow-zones and in neighbourhoods of the zeros of the relevant Airy functions, where modifications like (4.33) are valid.

The relations (4.32) have an additional use. The formulae such as (4.27) for the $P_{\nu r}$ involve constants $c_{\nu}$ which may be difficult to calculate; but from (4.32) we see that the evaluation in this way of one of the $P_{\nu r}$ will suffice,

(8) Something more than merely equating coefficients in (4.30), (4.31) is required to get (4.32), since in (4.30) the $O$ 's are not constants.

(7) The fact about $\mathrm{Ai}^{\prime}$ becomes relevant in $\$ 4.7$.

(8) These facts follow, by means of (4.22), (4.23), from the simpler facts concerning $L$ functions. The zero line of $L(\nu v)$ is arg $v=3 \pi / 2$; the large zeros are spaced at intervals $\pi / \nu$, approximately; the neighbourhoods corresponding to those defined for the Airy functions are all of radius $1 / \nu$ roughly; and therein the three $L$-functions are less than $2^{1 / 2} \pi$. 
provided the ratios of the $Q_{v r}$ are known. Inversely, if closed formulae for the ratios of the $Q_{\nu r}$ are not known (as for Mathieu's equation, for example), we can from (4.32) obtain approximations to them (for large $\nu$ ), using the $P_{\nu r}$ as found from (4.27) and similar equations for $P_{\nu 2}, P_{\nu 3}$.

The real case. Suppose that the differential equation (3.3) has, like all familiar ones, real coefficients. Then $Y_{\nu 1}, Q_{\nu 1}, P_{\nu 1}$ will be real and $Y_{\nu 2}, Q_{\nu 2}, P_{\nu 2}$ conjugate imaginary to $Y_{\nu 3}, Q_{\nu 3}, P_{\nu 3}$. From (4.32) it follows then that $\arg \left(Q_{\nu 2} / P_{\nu 2}\right)=-\arg \left(Q_{\nu 3} / P_{\nu 3}\right)= \pm \pi / 2$; so (assuming the ratios of the $Q_{\nu r}$ known) all the $P_{\nu r}$ will be known provided we can find $\left|P_{\nu 2}\right|$ and the sign of $P_{\nu 1}$. This case arises for the hypergeometric functions of Example 3, $\$ 2$.

The special real case. Suppose, in addition, that a single one of the infinite sectors of $\mathcal{D}_{z}$ includes the three rays arg $z=\pi, \pm 2 \pi / 3$; this occurs in Example 2 of $\$ 2$. Then a single formula (3.24) for $\phi$ is valid in the whole sector (provided continuations of $\phi^{3 / 2}, z^{1 / 2}$ across arg $z=\pi$ are taken), and in $\S 3.5$ it has been shown that $c_{\nu}$ is pure-imaginary. Hence the analogue of (4.27) will give $\left|P_{\nu 2}\right|$ without our knowing the value of $c_{\nu}$, and the explicit determination of all the $P_{v r}$ is easy.

4.6. Asymptotic formulae when $\nu$ is unreal. The assumption that $\nu$ is real positive has, in the work of $\S \S 4.2-4.5$, been essential only as regards the conditions defining Regions I-III, for example, (4.13), (4.15), and in the short cuts just explained for finding the $P_{\nu r}$. If we abandon this assumption, and suppose $\arg \nu$ to rotate through a certain angle, it is clear that we must take the "free" boundaries of Regions I-III, on the $v$-surface (that is, those boundaries which are not also boundaries of $\mathcal{D}_{v, v}$ ) to rotate through an equal angle in the opposite sense. The formula (4.26) will therefore remain valid in some part of $\mathcal{D}_{v, v}$ until the sector, centred on the ray arg $v=0$, into which we have integrated to prove it, gets engulfed in a shadow-zone; and this cannot happen until $\arg \nu$ is outside the limits $\pm \pi / 2$; while in Examples 2, 3, where the sector extends over $|\arg v| \leqq \pi / 2-\epsilon$, it will not happen until $|\arg \nu|$ $=\pi-\epsilon$.

Moreover (4.27) gives $P_{\nu 1}$ as an analytic function of $\nu$, so long as the said sector is not engulfed, so values of the $P_{\nu r}$ found by the short cut when $\nu$ is real can be extended analytically to all arg $\nu$ for which (4.26) is valid.

It is to be noted that, since $\nu$ is involved in (3.3) only as $\nu^{2}$, we can restrict attention to cases where $|\arg \nu| \leqq \pi / 2$, without essential loss. To summarise:

Theorem. If $\mid$ arg $\nu \mid \leqq \pi / 2$, the asymptotic formulae (4.26), (4.28) with modification such as (4.33) near the zeros of the relevant functions hold uniformly in $\mathcal{D}_{\zeta, \nu}$ apart from certain shadow-zones. For these formulae, the relation $\zeta=\phi(z)$ has been defined in $\$ 3.2$, and depends upon the integer $n$. For (4.26) a shadow-zone is one which, when mapped by $v=2 \zeta^{3 / 2} / 3$, cannot be joined to $v$ $=+\infty$ by a path within $\mathcal{D}_{v, v}$ whose slope (towards $+\infty$ ) is everywhere between $-\pi / 2-\arg \nu, \pi / 2-\arg \nu$ inclusive. For (4.28) the shadow-zones are similarly defined in relation to $v=\infty e^{\pi i}, \infty e^{-\pi i}$ respectively. 
A formula to replace (4.26) in a shadow-zone can be obtained by an evident modification of the procedure of $\$ 4.2$. From examples (see $\$ 4.9$ ) it appears that the free boundaries of such zones are effectively genuine boundaries for (4.26); they can always be pushed back a little, at the expense of increasing the constant of the error-term; but parallel to them are transition-lines, on crossing which the asymptotic equality of $P_{\nu 1} Y_{\nu 1}(z)\left(\phi^{\prime}(z) / 2\right)^{1 / 2}$ and $\pi \nu^{1 / 6} \mathrm{Ai}\left(\nu^{2 / 3 \zeta}\right)$ ceases.

4.7. Asymptotic series formulae. In (4.26) we have $\zeta=z+\sum_{1}^{\infty} \nu^{-2 r} \phi_{r}(z)$, $=z+\nu^{-2} h$, say. Let us expand $\mathrm{Ai}\left(\nu^{2 / 3} \zeta\right)$ in powers of $\nu^{-2} h$. Writing $\mathrm{Ai}\left(\nu^{2 / 3} z\right)$ $=f(z)$, we have

$$
\begin{aligned}
\operatorname{Ai}\left\{\nu^{2 / 3}\left(z+\nu^{-2} h\right)\right\}= & f\left(z+\nu^{-2} h\right)=f(z)+\nu^{-2} h \frac{d f(z)}{d z}+\cdots \\
& +\frac{\nu^{-4 n} h^{2 n}}{(2 n) !} f^{(2 n)}(z)+\frac{h^{2 n+1}}{(2 n+1) !} R,
\end{aligned}
$$

where $|R|$ is less than the greatest value of $\left|\nu^{-4 n-2} f^{(2 n+1)}(t)\right|$ on the segment from $t=z$ to $z+\nu^{-2} h$. We note from (3.23) that $z$ bounded gives $h$ bounded.

Now the Airy equation is $d^{2} f / d z^{2}=-\nu^{2} z f$, whence $d^{3} f / d z^{3}=-\nu^{2} f-\nu^{2} z d f / d z$, $f^{(4)}=\nu^{4} z^{2} f-2 \nu^{2} d f / d z$, and so on; $f^{(r)}$ has the form $M_{r} f+N_{r} d f / d z$, where $M_{r}, N_{r}$ are polynomials in $\nu, z$, involving only even powers of $\nu ; M_{r}$ is of degree at most $r$ in $\nu$, and $N_{r}$ at most $r-1$. Since $h$ is a power series in $\nu^{-2}, \nu^{-2 r} h^{r} f^{(r)}(z)$ takes the form $P_{r} f+Q_{r} d f / d z$, where $P_{r}, Q_{r}$ are power series in $\nu^{-2}$ beginning with terms in $\nu^{-r}, \nu^{-r-1}$. The coefficients in these series are polynomials in $z$ and the $\phi_{r}(z)$; and if we restrict $z$ to be bounded, the set of terms whose degree in $\nu^{-2}$ exceeds $n$ is uniformly $O\left(\nu^{-2 n-2}\right)$. Hence we obtain the form

$$
\begin{aligned}
\operatorname{Ai}\left(\nu^{2 / 3} \zeta\right)= & f(z)\left\{1+a_{2} \nu^{-2}+\cdots+a_{2 n} \nu^{-2 n}+O\left(\nu^{-2 n-2}\right)\right\} \\
& +d f(z) / d z\left\{a_{1} \nu^{-2}+\cdots+a_{2 n-1} \nu^{-2 n}+O\left(\nu^{-2 n-2}\right)\right\} \\
& +\frac{h^{2 n+1}}{(2 n+1) !} R .
\end{aligned}
$$

Now (using $t$ as current variable),

$$
\begin{aligned}
\left|f^{(r)}(t)\right|^{2} & =\left|M_{r} f+N_{r} d f / d t\right|^{2} \\
& \leqq\left(\left|M_{r}\right|^{2}+\left|\nu N_{r}\right|^{2}\right)\left(|f|^{2}+\left|\nu^{-1} d f / d t\right|^{2}\right) .
\end{aligned}
$$

If $s$ denotes arc-length on the straight segment from $t=z$ to $z+\nu^{-2} h$,

$$
\begin{gathered}
\left.\left|\frac{d}{d s}\right| f\right|^{2}|\leqq| \frac{d f^{2}}{d t}|=| 2 f d f / d t \mid \\
\left.\left|\frac{d}{d s}\right| \frac{d f / d t}{\nu}\right|^{2}|\leqq| \frac{2}{\nu^{2}} \frac{d f}{d t} \frac{d^{2} f}{d t^{2}}|=| 2 t f \frac{d f}{d t} \mid,
\end{gathered}
$$


and hence

$$
\begin{aligned}
\left|\frac{d}{d s}\left(|f|^{2}+\left|\frac{d f / d t}{\nu}\right|^{2}\right)\right| & \leqq(1+|t|)|2 f d f / d t| \\
& \leqq|\nu|(1+|t|)\left(|f|^{2}+\left|\frac{d f / d t}{\nu}\right|^{2}\right) .
\end{aligned}
$$

Hence for $t$ on the segment from $z$ to $z+\nu^{-2} h$,

$$
\begin{aligned}
\frac{|f|^{2}+\left|\nu^{-1} d f / d t\right|^{2}}{\left(|f|^{2}+\left|\nu^{-1} d f / d t\right|^{2}\right)_{t=z}} & \leqq \exp \left\{\left|\nu^{-2} h\right| \cdot|\nu|\left(1+|z|+\left|\nu^{-2} h\right|\right)\right\} \\
& =1+O\left(\nu^{-1}\right),
\end{aligned}
$$

since $z$ bounded implies that $h$ is bounded. Since $|R|$ is less than $|\nu|^{-4 n-2}\left|f^{(2 n+1)}(t)\right|_{\max }$, and $M_{2 n+1}, \nu N_{2 n+1}$ are $O\left(\nu^{2 n+1}\right),(4.35)$ gives therefore

$$
|R| \leqq A_{n}|\nu|^{-2 n-1}\left(|f(z)|^{2}+\left|\frac{d f(z)}{d z} / \nu\right|^{2}\right)^{1 / 2}
$$

and the remainder-term in (4.34) is of this same order since $h$ is bounded. We can write this remainder as $f(z) \cdot O\left(\nu^{-2 n-1}\right)$ or $d f(z) / d z \cdot O\left(\nu^{-2 n-2}\right)$, according as $|f(z)|$ does or does not exceed $\left|\nu^{-1} d f(z) / d z\right|$.

Hence in (4.34) the term in $R$ can be struck out, provided we write $O\left(\nu^{-2 n-1}\right)$ instead of $O\left(\nu^{-2 n-2}\right)$ in the coefficient of $f(z)$.

Substitute this expansion in (4.26) and incorporate with it the expansion of $\left(\phi^{\prime}(z)\right)^{1 / 2}=\left(1+\nu^{-2} \phi_{1}{ }^{\prime}(z)+\cdots\right)^{1 / 2}$. Putting finally $f(z)=\operatorname{Ai}\left(\nu^{2 / 3} z\right), d f(z) / d z$ $=\nu^{2 / 3} \mathrm{Ai}^{\prime}\left(\nu^{2 / 3} z\right)$, we obtain the form

$$
\begin{aligned}
P_{\nu 1} Y_{\nu 1}(z)=\pi 2^{1 / 2} \cdot \nu^{1 / 6} \operatorname{Ai}\left(\nu^{2 / 3} z\right)\left\{1+\nu^{-2} q_{2}(z)\right. & +\cdots \\
& \left.+\nu^{-2 n} q_{2 n}(z)+O\left(\nu^{-2 n-1}\right)\right\} \\
+\pi 2^{1 / 2} \cdot z^{5 / 6} \operatorname{Ai}^{\prime}\left(\nu^{2 / 3} v\right)\left\{\nu^{-2} q_{1}(z)\right. & +\cdots \\
& \left.+\nu^{-2 n} q_{2 n-1}(z)+O\left(\nu^{-2 n-2}\right)\right\} .
\end{aligned}
$$

Here $q_{1}, \cdots, q_{2 n}$ are polynomials in $z, \phi_{1}, \cdots, \phi_{n}, \phi_{1}^{\prime}, \cdots, \phi_{n}^{\prime}$; and these (in contrast to $\phi_{n+1}, \cdots$ ) are the $\phi$ 's which were determined from (3.11). Hence if the work, from $\$ 3.2$ onwards, be repeated with $n$ replaced by a larger integer, the expansion corresponding to (4.36) will coincide with (4.36) up to the terms in $\nu^{-2 n}$; and in analogy with familiar asymptotic series we can write

$$
P_{\nu 1} Y_{\nu 1}(z) \sim \pi 2^{1 / 2} \cdot \nu^{1 / 6} \mathrm{Ai}\left(\nu^{2 / 3} z\right)\left\{1+\sum_{1}^{\infty} \nu^{-2 r} q_{2 r}(z)\right\}
$$

$$
+\pi 2^{1 / 2} \cdot \nu^{5 / 6} \operatorname{Ai}^{\prime}\left(\nu^{2 / 3} z\right) \cdot \sum_{1}^{\infty} \nu^{-2 r} q_{2 r-1}(z)
$$


It follows also that in the first $\{\cdots\}$ of $(4.36)$, the remainder must be $O\left(\nu^{-2 n-2}\right)$.

The expansion (4.36) is valid provided that (4.26) is valid and $|z|$ is bounded. These conditions exclude neighbourhoods of the zeros $\zeta$ of $\mathrm{Ai}\left(\nu^{2 / 3} \zeta\right)$, whose radius is order $\nu^{-1} \zeta^{-1 / 2}$. Since $z=\zeta+O\left(\nu^{-2} \zeta\right)$, these coincide with neighbourhoods, of similar radius, of the zeros of $\mathrm{Ai}\left(\nu^{2 / 3} z\right)$; and $\mathrm{Ai}^{\prime}\left(\nu^{2 / 3} z\right)$ does not vanish in them. So from the maximum modulus principle, (4.36) is valid throughout these neighbourhoods, that is, (4.36) is valid under the conditions that $z$ is in $\mathcal{D}_{z}$ but not in a shadow-zone, and that $|z|$ is bounded.

There are, of course, similar formulae for $Y_{\nu 2}, Y_{\nu 3}$, and the coefficients $q_{r}$ are the same in all of them.

If we exclude suitable neighbourhoods of the zeros of $\mathrm{Ai}\left(\nu^{2 / 3} z\right)$, $\mathrm{Ai}^{\prime}\left(\nu^{2 / 3} z\right) / \mathrm{Ai}\left(\nu^{2 / 3} z\right)$ is bounded when $\nu^{2 / 3} z$ is bounded, but is $O\left(\nu^{1 / 3} z^{1 / 2}\right)$ when $\nu^{2 / 3} z$ is large. Hence in the two cases, the ratio of the second to the first term on the right of $(4.36)$ is $O\left(\nu^{-4 / 3}\right), O\left(\nu^{-1}\right)$ respectively $\left.{ }^{9}\right)$.

4.8. Approximation to Bessel functions of large order. The starting point is the differential equation (2.7), with the solution

$$
w=C s^{1 / 2} J_{\nu}\left\{\lambda\left(1-s^{2}\right)^{1 / 2}\right\}+D s^{1 / 2} Y_{\nu}\left\{\lambda\left(1-s^{2}\right)^{1 / 2}\right\} .
$$

The case usually considered is $\lambda=\nu$, but useful generality is gained by taking

$$
\lambda^{2}-\nu^{2}=\gamma_{0}+\gamma_{1} \nu^{-2}+\gamma_{2} \nu^{-4}+\cdots,
$$

a convergent series with real coefficients. The corresponding equation (3.3) of canonic form $\mathrm{A}$ is obtained by putting $u=2 z^{3 / 2} / 3, w=y z^{1 / 4}$, so from (2.9)

$$
\begin{array}{ll}
g_{0}=z\left\{\left(\frac{1}{s^{2}}-1\right)\left(\frac{5}{4 s^{4}}-\frac{1}{4 s^{2}}+\gamma_{0}\right)-\frac{5}{36 u^{2}}\right\}, & \\
g_{r}=\gamma_{r} z\left(\frac{1}{s^{2}}-1\right) & (r \geqq 1),
\end{array}
$$

where

$$
z=(3 u / 2)^{2 / 3}, \quad u=\operatorname{arctanh} s-s .
$$

The domain $\mathscr{D}_{z}$ is shown (apart from the excluded neighbourhoods of the two cuts) in Fig. 1 (iv), and for the values of $\alpha_{\nu}, \beta_{\nu}$ in (3.7) we have, from (2.11)

(9) Tricomi [5] has given a formula $\pi J_{\nu}\left\{\nu+t(\nu / 6)^{1 / 3}\right\}=(6 / \nu)^{1 / 3} A_{1}(t)-(1 / 10 \nu)\left\{3 t^{2} A_{1}{ }^{\prime}(t)\right.$ $\left.+2 t A_{1}(t)\right\}+O\left(\nu^{-5 / 3}\right)$, where $A_{1}(t)=3^{-1 / 3} \pi \mathrm{Ai}\left(-3^{1 / 3} t\right)$. The connection with that case of (4.36) which corresponds to $(4.46)_{1}$, below, is established by taking $\nu+t(\nu / 6)^{1 / 3}=\nu\left(1-s^{2}\right)^{1 / 2}$ and $z=2^{-2 / 3} s^{2}\left(1+(2 / 5) s^{2}+\cdots\right)$, which give $\nu^{2 / 3} z=-3^{-1 / 3} t+3 / 10 \cdot 18^{-1 / 3} \nu^{-2 / 3} t^{2}+\cdots$. Tricomi's two explicit terms arise then by expanding in (4.36) the principal term on the right and a factor $z^{1 / 4} s^{-1 / 2}$ which occurs on the left, in accordance with (4.45); the next term in (4.36), namely the one in $q_{1}$, is represented by Tricomi's error-term. His approximation is uniform only when $t$ is bounded. 
and (2.12):

$$
\begin{array}{ll}
\text { for }|\arg z| \leqq \pi / 3-\epsilon, & \alpha_{\nu}=\beta_{\nu}=0, \\
\text { for }|\arg (-z)| \leqq 2 \pi / 3, & \alpha_{\nu}=\nu^{2}-\lambda^{2}, \quad \beta_{\nu}=0 .
\end{array}
$$

The integrations whereby $\phi_{1}, \phi_{2}, \cdots$ are determined from (3.11) come out rationally in $z^{1 / 2}$ and the dependent parameters $u, s\left({ }^{10}\right)$, for example

$$
\begin{aligned}
\phi_{1}= & \frac{1}{z^{1 / 2}}\left\{-\frac{5}{72 u}+\frac{5}{24 s^{3}}-\frac{1}{8 s}-\frac{1}{2} \gamma_{0} s\right\} \\
= & \frac{1}{z^{1 / 2}}\left\{-\frac{5}{72 u}+f_{1}(s)\right\}, \text { say, } \\
\phi_{2}= & \frac{1}{z^{1 / 2}}\left\{-\frac{10}{243 u^{3}}+\frac{5 f_{1}(s)}{54 u^{2}}-\frac{f_{1}^{2}(s)}{6 u}+f_{2}(s)\right\}, \\
f_{2}(s)= & \frac{1105}{1152 s^{9}}-\frac{221}{128 s^{7}}+\left(\frac{531}{640}+\frac{5 \gamma_{0}}{16}\right) \frac{1}{s^{5}}-\left(\frac{25}{384}+\frac{3 \gamma_{0}}{8}\right) \frac{1}{s^{3}} \\
& +\left(\frac{\gamma_{0}}{16}+\frac{\gamma_{0}^{2}}{8}\right) \frac{1}{s}+\left(\frac{\gamma_{0}^{2}}{8}-\frac{\gamma_{1}}{2}\right) s .
\end{aligned}
$$

The regularity of $\phi_{1}, \phi_{2}, \cdots$ at $z=0$ is verified when we expand in ascending powers of $s$ by use of $u^{-1}=3 s^{-3}\left(1+3 s^{2} / 5+3 s^{4} / 7+\cdots\right)^{-1}$. This gives, for example

$$
\begin{aligned}
\phi_{1}=\left(\frac{1}{70}-\frac{\gamma_{0}}{2}\right) \frac{s}{z^{1 / 2}}+\frac{10^{-4}}{z^{1 / 2}}\left(7.301587 s^{3}\right. & +7.05257 s^{5} \\
& \left.+4.8966 s^{7}+3.244 s^{9}+\cdots\right),
\end{aligned}
$$

where

$$
z^{1 / 2}=2^{-1 / 3} s\left(1+\frac{3}{5} s^{2}+\frac{3}{7} s^{4}+\cdots\right)^{1 / 3}=s P\left(s^{2}\right)
$$

If, in using (4.46) below, we restrict $s$ to be bounded from 1 and $\infty-$ which covers the most important cases-it is sufficient to calculate $\phi_{1}, \cdots, \phi_{n}$; the complete series $\zeta=z+\sum_{1}^{\infty} \nu^{-2 r} \phi_{r}$, found by solving (3.15), is needed only to secure uniformity up to $s=1, \infty$.

For $Y_{\nu 1}, Y_{\nu 2}, Y_{\nu 3}$ in (4.26), (4.28) we are to pick standard functions $z^{-1 / 4} w$,

(10) The proof is omitted. The underlying fact is that (4.26) must give an elementary asymptotic expansion for $Y_{\nu 1}(z)$-in the present case Debye's "A" series [6]-when we substitute the corresponding expansion of the Airy function; and by reversing the argument we can construct $\zeta=\phi(z)$ from the two elementary asymptotic expansions. 
where $w$ has the form (4.37), which are zero respectively for $z=+\infty, \omega \infty$, $\omega^{2} \infty$ when $\nu$ is real positive. To these values of $z$ correspond, in accordance with Fig. 1, $\left(1-s^{2}\right)^{1 / 2}=0,-i \infty, i \infty$ respectively. Hence we take

$$
\left\{Y_{\nu 1}, Y_{\nu 2}, Y_{\nu 3}\right\}=z^{-1 / 4} s^{1 / 2}\left\{J_{\nu},-2^{-1} H_{\nu}^{(2)},-2^{-1} H_{\nu}^{(1)}\right\}\left(\lambda\left(1-s^{2}\right)^{1 / 2}\right),
$$

so that in (4.29), $Q_{\nu 1}=Q_{\nu 2}=Q_{\nu 3}=1$. For the evaluation of the $P_{\nu r}$ we have the "special real case" noted at the end of $\$ 4.5$, so that the analogue of $(4.27)$ for $P_{\nu 2}$ gives, on reference to (4.41),

$$
\left|P_{\nu 2}\right|=\lim _{z \rightarrow \infty \infty} \frac{(2 \pi)^{1 / 2} \exp \left(2 \lambda z^{3 / 2} / 3\right)}{(\lambda / \nu)^{1 / 2} \cdot\left|s^{1 / 2} H_{\nu}^{(2)}\left\{\lambda\left(1-s^{2}\right)^{1 / 2}\right\}\right|},
$$

provided $\nu$ is real positive. Now from (4.40) we have for $z \sim \omega \infty, 2 z^{3 / 2} / 3=u$ $=-s+\pi i / 2+O\left(s^{-1}\right)$, and by the elementary asymptotic formula for $H_{\nu}^{(2)}(\nu$ being fixed)

$$
\begin{aligned}
\left|s^{1 / 2} H_{\nu}^{(2)}\left\{\lambda\left(1-s^{2}\right)^{1 / 2}\right\}\right| & \sim\left|\left(\frac{2 s}{\pi \lambda}\right)^{1 / 2} \cdot \frac{\exp \left\{-i \lambda\left(1-s^{2}\right)^{1 / 2}\right\}}{\left(1-s^{2}\right)^{1 / 4}}\right| \\
& \sim\left|\left(\frac{2}{\pi \lambda}\right)^{1 / 2} e^{-\lambda s}\right| \sim\left|\left(\frac{2}{\pi \lambda}\right)^{1 / 2} \exp \left(\frac{2}{3} \lambda z^{3 / 2}\right)\right| .
\end{aligned}
$$

Hence $\left|P_{\nu 2}\right|=\pi \nu^{1 / 2}$, and since $P_{\nu 1}$ is clearly positive, (4.32) gives $P_{\nu 2}=i \pi \nu^{1 / 2}$, $P_{\nu 3}=-i \pi \nu^{1 / 2}, P_{\nu 1}=\pi \nu^{1 / 2}$. By analytic continuation, these values will be valid for those complex $\nu$ for which (4.46) below are valid.

Hence the formulae (4.26), (4.28) are equivalent to

$$
\begin{gathered}
J_{\nu}\left\{\lambda\left(1-s^{2}\right)^{1 / 2}\right\}=\left(\frac{2}{\phi^{\prime}(z)}\right)^{1 / 2} \cdot \frac{z^{1 / 4} \mathrm{Ai}\left(\nu^{2 / 3} \zeta\right)}{\nu^{1 / 3} s^{1 / 2}}\left\{1+O\left(\nu^{-2 n-1}\right)\right\} \\
2^{-1} H_{\nu}^{(2)}\left\{\lambda\left(1-s^{2}\right)^{1 / 2}\right\}=-\left(\frac{2}{\phi^{\prime}(z)}\right)^{1 / 2} \cdot \frac{z^{1 / 4} \omega^{2} \mathrm{Ai}\left(\nu^{2 / 3} \omega^{2} \zeta\right)}{\nu^{1 / 3} s^{1 / 2}}\left\{1+O\left(\nu^{-2 n-1}\right)\right\} \\
2^{-1} H_{\nu}^{(1)}\left\{\lambda\left(1-s^{2}\right)^{1 / 2}\right\}=-\left(\frac{2}{\phi^{\prime}(z)}\right)^{1 / 2} \cdot \frac{z^{1 / 4} \omega \mathrm{Ai}\left(\nu^{2 / 3} \omega \zeta\right)}{\nu^{1 / 3} s^{1 / 2}}\left\{1+O\left(\nu^{-2 n-1}\right)\right\}
\end{gathered}
$$

where $\zeta=\phi(z)=z+\sum_{1}^{\infty} \nu^{-2 r} \phi_{r}(z)$; and in consequence of $(4.40), z^{1 / 4} / s^{1 / 2}$ is regular at $s=0$ or $z=0$. From the last two we obtain

$$
Y_{\nu}\left\{\lambda\left(1-s^{2}\right)^{1 / 2}\right\}=-\left(\frac{2}{\phi^{\prime}(z)}\right)^{1 / 2} \cdot \frac{z^{1 / 4} \mathrm{Bi}\left(\nu^{2 / 3} \zeta\right)}{\nu^{1 / 3} s^{1 / 2}}\left\{1+O\left(\nu^{-2 n-1}\right)\right\},
$$

valid except near the zeros of the function; $\mathrm{Bi}(x)=e^{\pi i / 6} \mathrm{Ai}(\omega x)+e^{-\pi i / 6} \mathrm{Ai}\left(\omega^{2} x\right)$ is the second Airy function.

According to the Theorem of $\$ 4.6$, each of $(4.46)$ is valid in that part of $\mathcal{D}_{\zeta, \nu}$ which is not covered by a shadow-zone, and $\mathcal{D}_{\zeta, \nu}$ is the map of $\mathcal{D}_{z}$, Fig. 1 
(iv), by $\zeta=\phi(z)$. For $\arg \nu=0$ there are no shadow-zones. Now let arg $\nu$ decrease towards $-\pi / 2$, and for definiteness consider the case of $J_{\nu}$. Then in $\mathcal{D}_{v, \nu}$ (which is almost coincident with $\mathcal{D}_{u}$, so that Fig. 1 (iii) can represent a part of it) a shadow-zone develops on the left-hand side of the cut from $i \pi$ to $i \infty$; its free boundary begins, say, at $v=i(\pi-\epsilon)$, and makes an angle $-\arg \nu$ with the imaginary axis; so that for $\arg \nu=-\pi / 2$ the free boundary is the ray $\arg (v-i \pi+i \epsilon)=\pi$. This is the only shadow-zone, since the conjugate cut $(-i \pi,-i \infty)$ casts a shadow for $\arg \nu>0$ but not for $\arg \nu<0$.

Now on account of continuation formulae such as $J_{\nu}\left(x e^{\pi i}\right)=e^{\nu \pi i} J_{\nu}(x)$ it is sufficient to confine $\left(1-s^{2}\right)^{1 / 2}$ to the positive half-plane (Fig. 1 (i)), and to this corresponds for $u$ the L-shaped region $A B D C$ and its conjugate. Since the boundary $A B$ of this is $\operatorname{Im} u=\pi / 2$ and since $v=u\left\{1+O\left(\nu^{-2}\right)\right\}$, the corresponding boundary in $\mathcal{D}_{v, \nu}$ is certainly below the ray arg $(v-i \pi+i \epsilon)=\pi$, except possibly where $|v|$ is large. But then (3.24), with the appropriate values $(4.41)_{2}$ of $\alpha_{\nu}, \beta_{\nu}$, gives $2 \zeta^{3 / 2} / 3=2(\lambda / \nu) z^{3 / 2} / 3+O\left(\nu^{-2}\right)$, that is, $v=(\lambda / \nu) u$ $+O\left(\nu^{-2}\right)$. From (4.38), $\lambda / \nu$ is a power series in $\nu^{-2}$ with real coefficients, so to a boundary point $u=\pi i / 2-x$ corresponds

$v-\pi i / 2+O\left(\nu^{-2}\right)=-x\left\{1+\nu^{-2} \Phi\left(\nu^{-2}\right)\right\} \quad(x$ real, $P$ with real coefficients $)$.

The corresponding formula giving the boundary of the shadow-zone is

$$
v-i \pi+i \epsilon=-x^{\prime}\{1-i \tan (\pi / 2+\arg \nu)\} \quad \text { ( } x^{\prime} \text { real). }
$$

For $\arg \nu=-\pi / 2$ the right-hand members are both real, so the boundary $A B$ is entirely below the shadow-boundary. For arg $\nu=-\pi / 2+\theta(\theta>0)$ the same is true; for on the right the real parts are equal when $x^{\prime}=x\left\{1+O\left(\nu^{-2}\right)\right\}$, and the imaginary parts are respectively $x^{\prime} \cdot O(|\nu|-2 \sin 2 \theta)$ and $x^{\prime} \tan \theta$, of which the latter is the greater.

For the shadow-zones for $H_{\nu}^{(1)}, H_{\nu}^{(2)}$ there is a corresponding property, so the asymptotic formulae (4.46), (4.47) hold uniformly for $|\arg \nu| \leqq \pi / 2$ and $\left|\arg \left(1-s^{2}\right)^{1 / 2}\right| \leqq \pi / 2$, except that in small neighbourhoods of the zeros of $\mathrm{Ai}\left(\nu^{2 / 3} \zeta\right)$, and so on, the remainder terms $O\left(\nu^{-2 n-1}\right)$ must be made additive with $z^{1 / 4} \mathrm{Ai}\left(\nu^{2 / 3} \zeta\right)$, and so on.

For numerical approximation to $J_{\nu}(x)$ when $\nu$ is large we can in (4.46) give $\lambda$ any value we please, provided it is near to $\nu$, and determine $s$ from $x=\lambda\left(1-s^{2}\right)^{1 / 2}$. The case where $\lambda$ is so chosen that to $z=0$ shall correspond $\zeta=\phi(z)=0$ has been tested over the range $0.3 \leqq\left(1-s^{2}\right)^{1 / 2} \leqq 10$. Taking the approximation correct to $O\left(\nu^{-2}\right)$, the largest proportional error found for $\nu \geqq 5$ was 1 in 30000 , occurring at $\left(1-s^{2}\right)^{1 / 2}=0.3, \nu=5$. At this point Debye's "A" series, to $O\left(\nu^{-2}\right)$, gives only 1 in 3000. Even for $\nu=2$ the largest error was only 1 in 3000 . The approximation correct to $O\left(\nu^{-4}\right)$ gives one extra correct figure at $\nu=2$, and two or more for $\nu \geqq 5$.

4.9. Bessel functions (continued). By using continuation formulae we can find the full range of $\arg \nu$ for which any one of (4.46) is valid, and at the same 
time verify that the exclusion of the shadow-zones is not merely sufficient for its truth, but is also necessary. For example, we have

$$
J_{\nu}(\nu x)=e^{-\nu \pi i} J_{\nu}\left(\nu x e^{\pi i}\right)=2^{-1} H_{\nu^{\prime}}^{(2)}\left(\nu^{\prime} x\right)+2^{-1} e^{-2 \nu \pi i} H_{\nu^{\prime}}^{(1)}\left(\nu^{\prime} x\right) \quad\left(\nu^{\prime}=\nu e^{\pi i}\right),
$$

and if we take $\left|\arg \left(1-s^{2}\right)^{1 / 2}\right| \leqq \pi / 2$ and $-3 \pi / 2 \leqq \arg \nu \leqq-\pi / 2$ we can substitute from (4.46) for the functions on the right. If for brevity we omit the error-terms, this gives

$$
\begin{aligned}
J_{\nu}\left\{\lambda\left(1-s^{2}\right)^{1 / 2}\right\} & \\
= & \left(\frac{2}{\phi^{\prime}(z)}\right)^{1 / 2} \cdot \frac{z^{1 / 4}}{\nu^{1 / 3} s^{1 / 2}}\left\{\mathrm{Ai}\left(\nu^{2 / 3} \zeta\right)+e^{-2 \nu \pi i} \omega^{2} \mathrm{Ai}\left(\nu^{2 / 3} \omega^{2} \zeta\right)\right\},
\end{aligned}
$$

and $(4.46)_{1}$ remains valid only so long as the first term is dominant. When $\nu^{2 / 3} \zeta$ is bounded it remains dominant for $-\pi / 2 \geqq \arg \nu>-\pi$. But when $\nu^{2 / 3} \zeta$ is large, and (for definiteness) $2 \pi / 3 \leqq \arg \zeta \leqq \pi$, we have, from (4.23) and(4.10),

$$
\frac{\operatorname{Ai}\left(\nu^{2 / 3} \zeta\right)}{\operatorname{Ai}\left(\nu^{2 / 3} \omega^{2} \zeta\right)} \sim i \omega^{2} e^{-2 \nu v} .
$$

From (4.48) we find for $J_{\nu}$ a string of zeros at

$$
\nu(v-\pi i)=\pi i / 4+r \pi i \quad(r \text { positive integral and large }),
$$

approximately; these zeros lie on a line $\arg (v-\pi i)=\pi / 2-\arg \nu$ which is parallel to the free boundary of the shadow-zone discussed in $\$ 4.8$, and just within it; and on crossing the line into the shadow-zone the second term on the right of (4.48) becomes dominant. Thus if $v=2 \zeta^{3 / 2} / 3$ and arg $v$ is between $\pi$ and $3 \pi / 2$, with $|\nu v|$ large, a necessary condition for $(4.46)_{1}$ is $\arg \nu>\pi / 2$ $-\arg (v-\pi i)$.

5. Bessel function approximations to hypergeometric functions. Since the transformation (3.1), which converts (as nearly as we please) the Airy equation (3.2) into the arbitrary form-A equation (3.3) is reversible when $\nu$ is sufficiently large, there is a similar transformation connecting any two form-A equations. Hence there are asymptotic formulae for the solutions of any such equation in terms of the solutions of any other such equation. In particular, there are approximations in terms of Bessel functions of large order; and these are "acceptable" approximations, since the properties of Bessel functions are well known and they are well tabulated.

When we leave the neighbourhood of the transition point $z=0$, the resemblance between different form-A equations may of course disappear; for instance the domains $\mathcal{D}_{z}$ attached to the three Examples of $\$ 2$ have different boundaries. In a given case, the choice of Bessel- rather than Airy-approximations will usually be suggested by features remote from the transition point; and we should not make this choice unless the $\mathcal{D}_{z}$ attached to the Bessel functions, Fig. 1 (iv), contains the $\mathcal{D}_{z}$ attached to the other equation, for 
example Fig. 2 (iv).

In the case of the hypergeometric functions of Example 3, Bessel-function approximations are evidently suitable. There is no space here to enter into their detailed development, but we may note their general form, and one or two interesting features.

5.1. The form-A equation corresponding to (2.17) satisfies the hypotheses of $\$ 3.1$, so the theorem stated in $\$ 4.6$ is applicable. Hence there are formulae of the forms (4.26), (4.28) for the hypergeometric functions, and if we eliminate the Airy functions between these and the Bessel-function formulae of $\$ 4.8$ we obtain the desired formulae. These formulae are however more directly obtained by transforming the form-B Bessel equation (2.7) into the hypergeometric equation (2.17), correct to an assigned order in $\nu^{-2}$. This may be conveniently done by using $\lambda$ in (27) as an adjustable parameter, to be so chosen that the transition point $s=0$ of the Bessel functions may coincide (to the desired order) with the transition point $t=0$ of the hypergeometric functions. For this we put

$$
\lambda^{2}-\nu^{2}=\gamma_{0}+\gamma_{1} \nu^{-2}+\cdots,
$$

and choose the real coefficients $\gamma_{0}, \gamma_{1}, \cdots$ suitably; the value of $\gamma_{0}$ is

$$
\gamma_{0}=\frac{1}{35}+\frac{101-158 \tau_{s}-39 \tau_{s}^{2}}{140\left(1-\tau_{s}\right)^{4 / 3}} .
$$

For the transformation formulae it is sufficient to have $s$ as a function of $t, \nu^{-2}$. We can find $s$ as an explicit function of $t, \nu^{-2}$ and a parameter $x$ related to $t$ by

$$
\operatorname{arctanh} x-x=\operatorname{arctanh} t-\tau_{s}^{-1 / 2} \operatorname{arctanh}\left(t \tau_{s}^{1 / 2}\right),
$$

an equation which has to be solved numerically for $x$ when $t$ is given.

From the general theory we know that there is asymptotic equality between solutions of the Bessel and hypergeometric equations which both vanish at $z=+\infty$, or at $z=\omega \infty$, or at $z=\omega^{2} \infty$. Hence we find

$$
\begin{gathered}
(1-\tau)^{\beta / 2} t^{1 / 2} \tau^{\nu / 2} F_{\nu}(\tau)\left(\psi^{\prime}\right)^{1 / 2}=E_{\nu}(2 \pi \nu s)^{1 / 2} \cdot J_{\nu}\left\{\lambda\left(1-s^{2}\right)^{1 / 2}\right\} \\
\cdot\left\{1+O\left(\nu^{-2 n-1}\right)\right\}, \\
\begin{array}{c}
(1-\tau)^{\beta / 2} t^{1 / 2}\left\{\frac{e^{\nu \pi i} \tau^{\nu / 2} F_{\nu}(\tau)}{i \sin \nu \pi}-\frac{\tau^{-\nu / 2} F_{-\nu}(\tau)}{i \pi h_{\nu}}\right\}\left(\psi^{\prime}\right)^{1 / 2} \\
=C_{\nu}(2 \pi \nu s)^{1 / 2} \cdot H_{\nu}^{(2)}\left\{\lambda\left(1-s^{2}\right)^{1 / 2}\right\} \cdot\left\{1+O\left(\nu^{-2 n-1}\right)\right\}, \\
(1-\tau)^{\beta / 2} t^{1 / 2}\left\{\frac{e^{-\nu \pi i} \tau^{\nu / 2} F_{\nu}(\tau)}{-i \sin \nu \pi}+\frac{\tau^{-\nu / 2} F_{-\nu}(\tau)}{i \pi h_{\nu}}\right\}\left(\psi^{\prime}\right)^{1 / 2} \\
=D_{\nu}(2 \pi \nu s)^{1 / 2} \cdot H_{\nu}^{(1)}\left\{\lambda\left(1-s^{2}\right)^{1 / 2}\right\} \cdot\left\{1+O\left(\nu^{-2 n-1}\right)\right\},
\end{array}
\end{gathered}
$$


where $s$ is a known function of $\tau, \nu$ and $n$. Here $C_{\nu}(2 \pi \nu)^{1 / 2}$, and so on, play the part of $P_{\nu r}$ in $\S 4, \psi^{\prime}$ plays the part of $\phi^{\prime}$ :

$$
\psi^{\prime}=\frac{2 \tau s^{2}}{t\left(s^{2}-1\right)} \frac{d s}{d \tau}
$$

and

$$
h_{\nu}=\frac{\Gamma\left(a_{\nu}\right) \Gamma\left(1+\nu-b_{\nu}\right)}{\Gamma\left(a_{\nu}-\nu\right) \Gamma\left(1-b_{\nu}\right) \Gamma(\nu) \Gamma(\nu+1)} .
$$

The equations (2.14) give $a_{\nu}, b_{\nu}$ as double-valued functions of $\nu$, and in (5.6) are to be taken the determinations for which $a_{\nu}$ is large positive and $b_{\nu}$ large negative when $\nu$ is large real positive. When $|\arg \nu| \leqq \pi / 2$ these formulae hold uniformly in the $\tau$-plane (Fig. 2 (i)) cut from 0 to $-\infty$, excluding a neighbourhood of the cut from 1 to $+\infty$ and neighbourhoods of the appropriate zeros.

5.2. Regarding the evaluation of $E_{\nu}, C_{\nu}, D_{\nu}$ we have the "real case" referred to at the end of $\$ 4.5$, but not the "special real case"; nevertheless it is possible to find them explicitly. For brevity, let us omit the remainderfactors. Then the argument from conjugacy gives $C_{\nu}=D_{\nu}=E_{\nu}$, and by eliminating $F_{\nu}(\tau)$ from (5.4) we obtain

$$
(1-\tau)^{\beta / 2} t^{1 / 2} \tau^{-\nu / 2} F_{-\nu}(\tau)\left(\psi^{\prime}\right)^{1 / 2}=\frac{\pi h_{\nu} E_{\nu}(2 \pi \nu s)^{1 / 2}}{\sin \nu \pi} J_{-\nu}\left\{\lambda\left(1-s^{2}\right)^{1 / 2}\right\} .
$$

Now (5.3), (5.7) are both valid for $|\arg \nu| \leqq \pi / 2$, so we can put $\nu=\rho e^{-i \pi / 2}$ in (5.3) and $\nu=\rho e^{i \pi / 2}$ in (5.7), where $\rho$ is large real positive, and thereby obtain two formulae which must coincide. Since $J_{-\nu}\left(x e^{-\pi i}\right)=e^{\nu \pi i} J_{-\nu}(x)$, and $\arg \nu=-\pi / 2$ gives $\sin \nu \pi=-i e^{\nu \pi i} / 2$, we thus obtain

$$
E_{-\nu}=2 \pi h_{\nu} E_{\nu}
$$

But from (5.6) we can deduce, for $|\arg \nu| \leqq \pi-\epsilon$, an asymptotic expansion of the form

$$
\log \left(2 \pi h_{\nu}\right) \sim c_{0} \nu+c_{1} \nu^{-1}+c_{2} \nu^{-3}+\cdots
$$

involving only odd powers; and by letting $\tau \rightarrow 0, s \rightarrow 1$ in (5.3) we obtain an asymptotic expansion in odd powers for $\log E_{\nu}$, which is valid at least for $|\arg \nu| \leqq \pi / 2$. Hence (5.8) gives, for $\arg \nu=-\pi / 2$ in the first instance,

$$
E_{\nu}=\left(2 \pi h_{\nu}\right)^{-1 / 2} \text {, }
$$

and, since this implies identity of the asymptotic expansions of the two members, it is valid for $|\arg \nu| \leqq \pi / 2$. This completes the desired evaluation.

5.3. The formulae (5.3) and (5.7) may be unified as follows: We can take (5.8) as defining $E_{-\nu}$ when $|\arg \nu| \leqq \pi / 2$, this being consistent with (5.8) and 
(5.10) at $\arg \nu= \pm \pi / 2$; then, for all large $\nu$ the asymptotic expansion for $\log E_{\nu}$, just referred to, is valid. Let us now put

$$
J_{\nu}(x)=\frac{1}{\Gamma(\nu+1)}\left(\frac{x}{2}\right)^{\nu} \Lambda_{\nu}(x), \quad J_{-\nu}(x)=\frac{\Gamma(\nu+1) \sin \nu \pi}{\nu \pi}\left(\frac{x}{2}\right)^{-\nu} \Lambda_{-\nu}(x),
$$

so that $\Lambda_{\nu}(x)$ is single-valued in $x$ and $\nu$, even in $x$, and meromorphic in $\nu$, with poles at $\nu=-1,-2, \cdots$. Then (5.3), (5.7) can be written

$$
\begin{aligned}
(1-\tau)^{\beta / 2} F_{\nu}(\tau) & =E_{\nu}\left[\frac{(2 \pi \nu)^{1 / 2}}{\Gamma(\nu+1)}\left(\frac{\nu}{e}\right)^{\nu}\left(\frac{\lambda}{\nu}\right)^{\nu}\right]\left\{\frac{e}{2}\left(\frac{1-s^{2}}{\tau}\right)^{1 / 2}\right\}^{\nu}\left(\frac{s}{t \psi^{\prime}}\right)^{1 / 2} \\
& \cdot \Lambda_{\nu}\left\{\lambda\left(1-s^{2}\right)^{1 / 2}\right\}\left\{1+O\left(\nu^{-2 n-1}\right)\right\}, \\
(1-\tau)^{\beta / 2} F_{-\nu}(\tau) & \\
= & E_{-\nu}\left[\frac{\Gamma(\nu+1)}{(2 \pi \nu)^{1 / 2}}\left(\frac{\nu}{e}\right)^{-\nu}\left(\frac{\lambda}{\nu}\right)^{-\nu}\right]\left\{\frac{e}{2}\left(\frac{1-s^{2}}{\tau}\right)^{1 / 2}\right\}^{-\nu}\left(\frac{s}{t \psi^{\prime}}\right)^{1 / 2} \\
& \cdot \Lambda_{-\nu}\left\{-\lambda\left(1-s^{2}\right)^{1 / 2}\right\}\left\{1+O\left(\nu^{-2 n-1}\right)\right\} .
\end{aligned}
$$

Since $\lambda / \nu$ is an even function of $\nu$ the two factors in square brackets have asymptotic expansions which are interchanged by reversing the sign of $\nu$; so since also $s, \psi^{\prime}$ are even functions of $\nu,(5.12)$ is derived from (5.11) by reversing the sign of $\nu$. Since both formulae are valid for $|\arg \nu| \leqq \pi / 2$, (5.11) is valid without restriction on arg $\nu$. The poles of $\Lambda_{\nu}$ cancel those of $F_{\nu},\left(1-s^{2}\right)^{1 / 2} / \tau$ is regular at $\tau=0, s=1$, and $\left(s / t \psi^{\prime}\right)^{1 / 2}$ is regular at $s=0, t=0$. Hence the cut from $\tau=0$ to $-\infty$ is unnecessary, and the only restriction upon $\tau$ is that it be excluded from a neighbourhood of the cut $(+1,+\infty)$ and from small neighbourhoods of the zeros of $F_{\nu}(\tau)$.

5.4. By an argument modelled on $\$ 4.7$ we can convert (5.3), (5.7) into the forms

$$
\begin{aligned}
(1-\tau)^{\beta / 2} t^{1 / 2} \tau^{\nu / 2} F_{\nu}(\tau) & \sim\left(\frac{\nu x}{h_{\nu}}\right)^{1 / 2}\left[J_{\nu}\left\{\nu\left(1-x^{2}\right)^{1 / 2}\right\}\left(1+q_{2} \nu^{-2}+q_{4} \nu^{-4}+\cdots\right)\right. \\
+ & \left.\left(1-x^{2}\right)^{1 / 2} J_{\nu}^{\prime}\left\{\nu\left(1-x^{2}\right)^{1 / 2}\right\}\left(q_{1} \nu^{-1}+q_{3^{2}}-3+\cdots\right)\right], \\
(1-\tau)^{\beta / 2} t^{1 / 2} \tau^{-\nu / 2} F_{-\nu}(\tau) & \sim \frac{\pi\left(\nu x h_{\nu}\right)^{1 / 2}}{\sin \nu \pi}\left[J_{-\nu}\left\{\nu\left(1-x^{2}\right)^{1 / 2}\right\}\left(1+q_{2} \nu^{-2}+\cdots\right)\right. \\
& \left.+\left(1-x^{2}\right)^{1 / 2} J_{-\nu}^{\prime}\left\{\nu\left(1-x^{2}\right)^{1 / 2}\right\}\left(q_{1} \nu^{-1}+\cdots\right)\right] .
\end{aligned}
$$

Here $x$ is determined by $\tau$ only by means of (5.2) and (2.15). The range of validity differs from that of (5.3), (5.7) in that a neighbourhood of $\tau=\infty$ must be excluded, but there is no need to exclude neighbourhoods of the zeros of $F_{v}, F_{-\nu}$. The coefficients $q_{1}, q_{2}, \cdots$ may be calculated, as explicit functions of $x$ and $t$, by elementary processes. 


\section{BIBLIOGRAPHY}

1. H. Jeffreys, Monthly Notices of the Royal Astronomical Society. Geophysical Supplement. vol. 2 (1928) pp. 101-111.

2. British Association Tables, Part Volume B, J. C. P. Miller, 1946.

3. R. E. Langer, Trans. Amer. Math. Soc. vol. 33 (1931) pp. 23-64 and vol. 34 (1932) pp. 447-480.

4. T. M. Cherry, J. London Math. Soc. vol. 24 (1949) pp. 121-130.

5. F. Tricomi, Sulle funzioni di Bessel di ordine e argomento pressoche uquali, Atti della Accademia delle Scienze di Torino vol. 83 (1947-1948).

6. G. N. Watson, Bessel functions, \$§8.4-8.42.

UNiversity of MELbourne, Melbourne, Australia. 